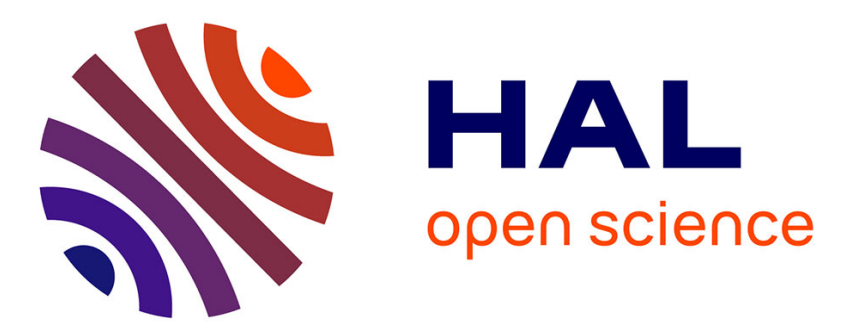

\title{
Study of vacancy-(H,B,C,N,O) clusters in Al using DFT and statistical approaches: Consequences on solubility of solutes
}

Damien Connétable, Matthieu David

\section{To cite this version:}

Damien Connétable, Matthieu David. Study of vacancy-(H,B,C,N,O) clusters in Al using DFT and statistical approaches: Consequences on solubility of solutes. Journal of Alloys and Compounds, 2018, 748, pp.12-25. 10.1016/j.jallcom.2018.03.081 . hal-01993617

\section{HAL Id: hal-01993617 https://hal.science/hal-01993617}

Submitted on 25 Jan 2019

HAL is a multi-disciplinary open access archive for the deposit and dissemination of scientific research documents, whether they are published or not. The documents may come from teaching and research institutions in France or abroad, or from public or private research centers.
L'archive ouverte pluridisciplinaire HAL, est destinée au dépôt et à la diffusion de documents scientifiques de niveau recherche, publiés ou non, émanant des établissements d'enseignement et de recherche français ou étrangers, des laboratoires publics ou privés. 


\section{Open Archive Toulouse Archive Ouverte (OATAO)}

OATAO is an open access repository that collects the work of Toulouse researchers and makes it freely available over the web where possible

This is an author's version published in: http://oatao.univ-toulouse.fr/21419

Official URL: https://doi.org/10.1016/j.jallcom.2018.03.081

\section{To cite this version:}

Connétable, Damien $\rightleftharpoons$ and David, Matthieu $(H, B, C, N, O)$ clusters in Al using DFT and statistical approaches: Consequences on solubility of solutes. (2018) Journal of Alloys and Compounds, 748. 12-25. ISSN 0925-8388

Any correspondence concerning this service should be sent to the repository administrator: tech-oatao@listes-diff.inp-toulouse.fr 


\title{
Study of vacancy-(H,B,C,N,O) clusters in Al using DFT and statistical approaches: Consequences on solubility of solutes
}

\author{
Damien Connétable*, Matthieu David \\ CIRIMAT UMR 5085, CNRS-INP-UPS, ENSIACET 4, allée Émile Monso, BP 44362, F-31030, Toulouse Cedex 4, France
}

A R T I C L E I N F O

Keywords:

Aluminum

Interstitial elements

DFT

Statistical approach

Clusters

\begin{abstract}
A B S T R A C T
This article is a study of the solubility of interstitial atoms in aluminum using a multi-scale approach. We focused on hydrogen, boron, carbon, nitrogen and oxygen atoms (labeled $X$ ). We studied isolated atoms as well as the possible formation of clusters with and without vacancies (V), i.e., $X_{m}, V X_{m}$ and $V_{2} X_{m}(m$ $\geq 0$ ). Formation and segregation energies are first obtained using first-principles calculations, subsequently, a statistical approach is employed in order to evaluate the concentration of impurities and defects according to the temperature. For instance, we find that, in solute solution, $\mathrm{H}, \mathrm{N}$ and $\mathrm{O}$ atoms prefer to be located in tetrahedral sites, and C and B atoms in octahedral sites. The chemical and energetic interactions between the interstitials, the metal and the vacancies are consequently presented and analyzed in detail. Results show that certain species prefer to interact with themselves, thus forming $X_{m}$ clusters, and others with vacancies, thus forming stable $\mathrm{VX}_{m}$ clusters in the metal. Using a statistical approach, we finally discuss the formation of clusters according to the temperature and the $X$ concentration. At low and intermediate temperatures (below $600 \mathrm{~K}$ ), we found that the atoms prefer to form clusters rather than stay isolated in aluminum. We show that $\mathrm{H}$ and $\mathrm{B}$ atoms are the only elements likely to increase vacancy concentration.
\end{abstract}

\section{Introduction}

It has been shown in earlier works [1-5] that the presence of interstitials, even in undetectable quantities, can have a significant impact on the concentration of defects (clusters, cavities, etc.) present in metals, especially on vacancy concentration. Therefore, the concentration of interstitial elements and point defects can be significantly different from what is expected with conventional laws (like Sieverts' law [6]). For instance, in the case of nickel [1,7], it has been shown that $\mathrm{O}$ and $\mathrm{H}$ atoms can form high concentrations of clusters $\left(V_{n} X_{m}\right.$, composed of $n V$ vacancies and $m \mathrm{X}$ atoms), especially at low and intermediate temperatures. In the case of iron, Schuler et al. [4] have shown that $\mathrm{C}$ atoms can form $\mathrm{V}_{2} \mathrm{C}_{2}$ clusters in a non-negligible concentration. The use of relevant multi-scale approaches has also clearly proved $[1,4,8]$ that DFT simulations alone are not able to analyze and interpret these mechanisms. It has been shown that the energy associated with cluster formation in the metal can strongly reduce the formation of vacancies, thus

\footnotetext{
* Corresponding author.

E-mail addresses: damien.connetable@ensiacet.fr (D. Connétable), matthieu. david@ensiacet.fr (M. David).
}

increasing the concentration of defects in the system, consequently enhancing the processes of diffusion of all species in the metal.

In the present work, we discuss the insertion of five interstitial elements in fcc-Al: hydrogen, boron, carbon, oxygen and nitrogen. Their interactions with vacancies and their capacity to form clusters in aluminum are thus discussed. The formation of $\mathrm{H}$ and He clusters in aluminum has been partly discussed in the literature: either only $\mathrm{VX}_{m}$ clusters were considered [5,8-10], or the multi-scale approach was not aimed at discussing the actual influence of cluster concentration on vacancy concentration [11]. In a recent work [12], diffusion mechanisms of interstitial elements were presented without discussing the interactions between $\mathrm{Al}$ and interstitials. This manuscript is aimed at filling these gaps as well as studying the case of other interstitials.

From an experimental standpoint, studies show that the maximum solubility of interstitial species in aluminum strongly depends on experimental conditions as well as on the specie. It has been shown that the limit of solubility is low in the case of $\mathrm{H}, \mathrm{C}, \mathrm{N}, \mathrm{O}$ and $\mathrm{B}$ atoms, even at high temperature. The content of nitrogen dissolved in the Al matrix is thus probably low. But according to Wriedt's [13] observations, this experimental data has little credibility. The exact $\mathrm{N}$ concentration is therefore unknown but can be 
considered relatively low. When the fugacity of $\mathrm{N}_{2}$ becomes too "high" (10-20 Pa at $933 \mathrm{~K}$ [13]), an AlN phase, with a wurtzite type structure, is formed. As for nitrogen, the Al-O phase diagram [14] is composed of only one intermediate structure between Al-fcc and $\mathrm{O}_{2}$ gas: alumina $\alpha-\mathrm{Al}_{2} \mathrm{O}_{3}$ (with many additional allotropic structures). The $O$ content in the metal is likewise supposed very low. The solid solubility of $\mathrm{C}$ atoms is also low, it is estimated at 400-800 appm [15]. However, Okamoto [16] explains that this concentration value is probably overestimated by at least one order of magnitude. In the case of hydrogen, the study of $\mathrm{Al}-\mathrm{H}$ system [17] also shows that hydrogen barely dissolves in aluminum: 15-20 appm near the melting point. Finally, the boron concentration is also low: a maximum solid solubility of $45 \mathrm{appm}$ of $\mathrm{B}$ in $\mathrm{Al}$ has been measured at $933 \mathrm{~K}$, see the Al-B system [18]. In summary, the concentration of interstitial elements can be considered ranging from 10 to $100 \mathrm{appm}$.

This work aims at evaluating solubility energies of these species and identifying the forms under which they are dissolved in the metal as a function of the temperature (T). The mechanisms of diffusion and the thermodynamics should vary depending on whether they are free or form clusters. As a first-order approximation, we used a multi-physics approach coupling first-principles simulations and a statistical model. At the atomic scale, simulations allow modeling free species $(X)$ and different types of clusters $\left(X_{m}\right.$, $\mathrm{VX}_{m}$ and $\mathrm{V}_{2} \mathrm{X}_{m}$ ) in the metal. It is not an exhaustive study, not all configurations were considered. However, the current work provides a broad overview of the interactions between $\mathrm{X}$ atoms and $\mathrm{Al}$. We calculated the formation energies of these clusters and analyzed their chemical properties. Subsequently, by using these accurate values in the statistical model, we computed the distribution and concentration of the different clusters as a function of $\mathrm{T}$ and the total concentration of interstitials in the metal.

The remainder of this paper is organized as follows. Section 2 summarizes the DFT parameters and briefly describes the model that was used. In part 3 , we discuss the insertion of the five species in the light of DFT results. The formation of different types of clusters is then presented (sections 4-6). We will conclude (section 7) with a discussion on the concentration and fraction of clusters in the metal using a statistical approach.

\section{Computational details}

First-principles calculations were performed with the density functional theory (DFT) using VASP (Vienna $a b$ initio simulation package [19]). We used the Perdew-Burke-Ernzerhof (PBE [20]) functional. Projector augmented wave (PAW) pseudo-potentials [21] were employed to describe atoms. As aluminum is not magnetic, the magnetic moment was not taken into account. In Appendix A, we report some results about several stable structures used hereinafter, especially reference states. Experimental structures are remarkably well reproduced by the DFT.

To study $V_{n} X_{m}$ clusters, simulations were carried out on a large super-cell ( $3 \times 3 \times 3$, that which corresponds to 108 atoms per unitcell) with full periodic boundary conditions. The inter-atomic forces were fully relaxed and all calculations were performed at zero pressure. We made sure that atomic forces were always smaller than $0.01 \mathrm{eV} / \AA$ on the atoms. The plane-wave cut-off energy was set to $600 \mathrm{eV}$, and $8 \times 8 \times 8$ Monkhorst-Pack mesh grids [22] were used to sample the first-Brillouin zones. With these criteria we obtain converged formation and segregation energies ( $<2-3 \mathrm{meV})$. To compute frequencies, we used the finite displacement method on super-cells. As a first-order approximation, only frequencies of $X$ atoms were computed, we thus neglect the effects of the insertion on $\mathrm{Al}$ frequencies (values reported in tables). However, due to the strong coupling between $\mathrm{Al}$ and $\mathrm{X}$ frequencies, we calculated the full inter-atomic force constants on $2 \times 2 \times 2$ super-cells, displacing only non-equivalent atoms along non-equivalent directions according to the symmetry of the system. The phonopy package [23] was used to generate finite displacements according to the symmetry of each structure, it was then used to analyze, plot vibrational properties (phonon dispersion curves and density of states, not shown here) and compute vibrational free energies.

To evaluate the distribution and concentration of clusters, we used an approach similar to the one described by Dome et al. [1]. For each type of cluster $i$ of $V_{p} X_{m}$ composed of $m X$ atoms and $p$ vacancies, its concentration $\left(C_{i}\left[V_{p} X_{m}\right]\right)$ is related to its formation energy $\left(H_{f}^{i}\left[V_{p} X_{m}\right]\right)$ and its number of equivalent configuration $\left(\mathscr{D}_{i}\left[V_{p} X_{m}\right]\right)$ :

$C_{i}\left[V_{p} X_{m}\right] \simeq \mathscr{D}_{i}\left[V_{p} X_{m}\right] \exp \left(-H_{f}^{i}\left[V_{p} X_{m}\right] / k_{B} T\right)$

The general formulation of the formation energy $\left(\mathrm{H}_{f}\right)$ of each $\mathrm{V}_{p} \mathrm{X}_{m}$ cluster is expressed by:

$H_{f}^{i}\left[V_{p} X_{m}\right]=E_{0}[(M-p) \cdot A l+m \cdot X]-E_{0}[M \cdot A l]+p \cdot \mu[A l]-m \cdot \mu[X]$

where $E_{o}[(M-p) \cdot A l+m . X]$ and $E_{o}[M . A l]$ are the DFT energies of the system with and without a cluster (composed of $M \mathrm{Al}$ atoms), respectively. The chemical potentials $(\mu)$ were taken equal to the energy per atom either of the perfect structure for $\mathrm{Al}\left(E_{o}[M . A l] / M\right)$ or of the reference state of $X$. In the tables, $H_{f}$ values were computed using $\mu[X]=E_{0}[$ ref $]$ (see Appendix A). To compute concentrations, the temperature $(T)$ and the chemical potential $-\mu[X]-$ (or the total concentration, $C_{\text {tot }}[X]$ ) are the free parameters of the equations.

The total vacancy concentration, $C_{t o t}[V]$ and $C_{t o t}[X]$ are thus given by:

$$
\left\{\begin{array}{l}
C_{\text {tot }}[V]=\sum_{p, m, j} p \cdot C_{j}\left[V_{p} X_{m}\right] \\
C_{\text {tot }}[X]=\sum_{p, m, j} m \cdot C_{j}\left[V_{p} X_{m}\right]
\end{array}\right.
$$

In the following discussions, we will also use the segregation energy of $X$ in $V_{p} X_{m}\left(E_{s e g}\left[V_{p} X_{m}\right]\right)$ expressed by:

$$
\begin{aligned}
E_{\text {seg }}\left[V_{p} X_{m}\right]= & E_{o}[(M-p) \cdot A l+m \cdot X]+E_{o}[M \cdot A l]-E_{o}[(M-p) \cdot A l \\
& +(m-1) \cdot X]-E_{o}[M \cdot A l+X]
\end{aligned}
$$

where $E_{o}[(M-p) \cdot A l+m \cdot X]$ and $E_{o}[(M-p) \cdot A l+(m-1) \cdot X]$ are the DFT energies of the $\mathrm{V}_{p} \mathrm{X}_{m}$ and $\mathrm{V}_{p} \mathrm{X}_{m-1}$ clusters, respectively. Segregation energy, not directly used in the thermodynamic model presented above, nevertheless allows us to provide information on atomic-scale processes. Indeed, it gives an idea of the affinity between a defect (here $\left.V_{p} X_{n-1}\right)$ and an isolated atom $(X)$, and the possibility that it is trapped. We will therefore give this quantity in addition to the formation energy.

\section{Ideal solubility}

From first-principles calculations, we first discuss the formation energies of $X$ in fcc-Al in the dilute limit, and analyze their relative stability. Three configurations were considered: the substituted, the tetrahedral ( $t$, in $8 c$ Wyckoff position) and the octahedral sites $(0,4 b)$. The formation energies and the zero-point energies (ZPE) 
Table 1

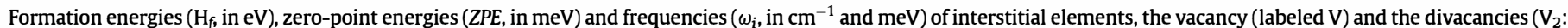

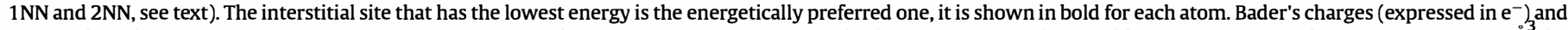

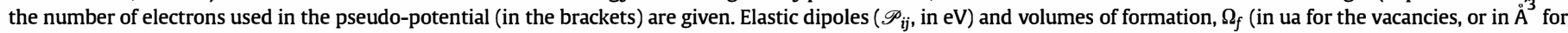
others) are also reported. In the case of $1 \mathrm{NN}$ and $2 \mathrm{NN}$ divacancies, there are two $\mathscr{P}_{i j}$ values and three for the $M$ configuration.

\begin{tabular}{|c|c|c|c|c|c|c|c|c|c|c|c|}
\hline & \multirow[t]{2}{*}{ site } & \multirow{2}{*}{$\frac{\mathrm{H}_{f}}{\mathrm{eV}}$} & \multirow{2}{*}{$\frac{\Delta E}{\mathrm{eV}}$} & \multirow{2}{*}{$\frac{\mathrm{ZPE}}{\mathrm{meV}}$} & \multicolumn{2}{|l|}{$\omega_{i}$} & \multirow{2}{*}{$\begin{array}{l}\text { charge } \\
\mathrm{e}^{-}\end{array}$} & \multicolumn{3}{|l|}{$\mathscr{P}_{i j}$} & \multirow[t]{2}{*}{$\Omega_{f}$} \\
\hline & & & & & $\mathrm{cm}^{-1}$ & $\mathrm{meV}$ & & $\mathrm{eV}$ & & & \\
\hline V & - & 0.632 & - & - & - & - & - & -2.5 & & & 0.65 \\
\hline \multirow[t]{2}{*}{$\mathrm{V}_{2}$} & $1 \mathrm{NN}$ & 1.324 & - & - & - & - & - & {$\left[\begin{array}{c}-4.9 \\
0 \\
0\end{array}\right.$} & $\begin{array}{c}0 \\
-4.9 \\
0\end{array}$ & $\left.\begin{array}{cc} & 0 \\
.9 & 0 \\
& -4.6\end{array}\right]$ & 1.71 \\
\hline & $2 \mathrm{NN}$ & 1.253 & - & - & - & - & - & {$\left[\begin{array}{c}-4.8 \\
0 \\
0\end{array}\right.$} & $\begin{array}{c}0 \\
-4.8 \\
0\end{array}$ & $\left.\begin{array}{cc}8 & 0 \\
8 & 0 \\
& -5.7\end{array}\right]$ & 1.77 \\
\hline \multirow[t]{4}{*}{$\mathrm{H}$} & sub & 1.806 & 1.089 & & & & unstable & & & & \\
\hline & $t$ & 0.717 & 0 & +13 & 853 & 106 & $2.1(1)$ & +2.1 & & & 4.8 \\
\hline & 0 & 0.823 & 0.106 & -68 & 420 & 52 & $2.3(1)$ & +1.2 & & & 3.0 \\
\hline & $M$ & 1.099 & 0.383 & & & & unstable & & & & \\
\hline \multirow[t]{4}{*}{ B } & sub & 2.399 & 1.170 & & & & unstable & & & & \\
\hline & $t$ & 2.413 & 1.184 & & unstable & & $5.3(3)$ & +8.6 & & & 18.8 \\
\hline & 0 & 1.229 & 0 & -33 & 528 & 65 & $6.3(3)$ & +5.1 & & & 11.1 \\
\hline & $M$ & 1.979 & 0.750 & & & & unstable & & & & \\
\hline \multirow[t]{4}{*}{ C } & sub & 4.075 & 2.753 & & & & unstable & & & & \\
\hline & $t$ & 1.691 & 0.369 & -66 & 572 & 71 & $6.6(4)$ & +6.6 & & & 14.5 \\
\hline & 0 & 1.322 & 0 & -94 & 425 & 53 & $7.2(4)$ & +2.8 & & & 6.4 \\
\hline & $M$ & 1.902 & 0.580 & -82 & $775 / 281 / 209$ & $96 / 34 / 26$ & $7.1(4)$ & {$\left[\begin{array}{cc}9.4 & 2 \\
2.7 & 9 \\
0 & \end{array}\right.$} & $\begin{array}{c}2.7 \\
9.4 \\
0\end{array}$ & $\left.\begin{array}{c}0 \\
0 \\
0.7\end{array}\right]$ & 12.3 \\
\hline \multirow[t]{4}{*}{$\mathbf{N}$} & sub & 3.040 & 3.944 & & & & unstable & & & & \\
\hline & $t$ & -0.904 & 0 & +27 & 550 & 68 & $7.3(5)$ & +4.8 & & & 10.6 \\
\hline & 0 & -0.056 & 0.850 & -29 & 253 & 31 & $7.7(5)$ & +2.0 & & & 4.6 \\
\hline & $M$ & 0.222 & 1.125 & & & & unstable & & & & \\
\hline \multirow[t]{4}{*}{0} & sub & 0.066 & 3.544 & & & & unstable & & & & \\
\hline & $t$ & -3.478 & 0 & +24 & 389 & 48 & $7.8(6)$ & +4.2 & & & 8.8 \\
\hline & 0 & -1.992 & 1.486 & & unstable & & $8.0(6)$ & +3.3 & & & 6.9 \\
\hline & $M$ & -2.311 & 1.167 & & & & unstable & & & & \\
\hline
\end{tabular}

are listed in Table 1. Within the harmonic approximation, the ZPE is expressed by:

$$
\begin{aligned}
Z P E= & \sum_{i, q} \hbar \omega_{i, q}[\text { metal }+\mathrm{X}] / 2-\sum_{i, q} \hbar \omega_{i, q}[\text { metal }] / 2 \\
& -\sum \hbar \omega^{\mathrm{ref}}[X] / 2
\end{aligned}
$$

where $\omega_{i, q}$ are the frequencies of $X$ atoms inside the metal and $\omega^{\text {ref }}[X]$ are the frequencies of the reference states: for $\mathrm{O}, \mathrm{N}$ and $\mathrm{H}$ atoms, we used the vibrations of $\mathrm{O}_{2}, \mathrm{~N}_{2}$ and $\mathrm{H}_{2}$ molecules respectively, in the case of carbon and boron, we used the diamond phase and the $\alpha$-B12 structure respectively as reference states (see Appendix A.1). The frequencies of $X$ in metal $\left(\omega_{i}\right)$, listed in Table 1 , have a degeneracy of three.

As seen in a previous work [12], all species are located in interstitial and not in substitution sites. The formation energy of the substituted sites is almost $1-3 \mathrm{eV}$ higher than the one of the other configurations, except for B atom, where the tetrahedral and substituted sites nearly have the same energy (see Table 1), same as in nickel [24]. However, frequency analysis shows that substituted positions are always unstable: there are always three imaginary frequencies for the $X$ atoms. The species move from the ideal position and form vacancy-specie clusters, as we will see below.

Contrary to nickel [7,24], in which the most stable positions for all interstitial species are the octahedral sites, the most stable configuration in $\mathrm{Al}$ depends on the atom.

In the case of $\mathrm{H}$ atoms, we agree with Wolverton [25] on the fact that, at low temperature, the $t$ site is the most stable configuration, when we take into account all relaxations, i.e., atomic and lattice relaxations and ZPE. The hydrogen solubility energy $(\simeq 0.73 \mathrm{eV})$ is in excellent agreement with earlier literature (see experimental and theoretical references cited by Nazarov et al. [8]). The difference in energy between both sites is however very small: taking into account the zero-point energy, it is equal approximately to $+25 \mathrm{meV}$. Similarly to hydrogen, we obtain that nitrogen is more stable in the tetrahedral positions than in the octahedral positions. However, the energy difference between both configurations $(+0.90 \mathrm{eV})$ is higher for hydrogen.

$O$ atoms prefer to be located in the $t$ sites, the difference in energy $(\Delta E)$ with the $o$ site is about $1.5 \mathrm{eV}$. Nevertheless, the analysis of $O$ frequencies in the octahedral sites shows that this configuration is unstable: three imaginary frequencies were obtained. We looked for stable intermediate configurations by moving oxygen from the ideal $o$ site. Nonetheless, the energy landscape seems to show that the only stable position is the tetrahedral site. If we move the 0 atom with a random displacement around the octahedral position, it moves towards the tetrahedral position. There is no additional intermediate site for $\mathrm{O}$ atoms.

For $C$ atoms, the energy of the $o$ site is significantly smaller than the one of the $t$ site, approximately $0.37 \mathrm{eV}$. The ZPE does not change the relative stability, but slightly corrects the solubility enthalpy. However, contrary to literature results regarding fcc structures (except the recent work of David [12]), we found another stable position in the fcc-lattice for carbon. This position, labeled $M$ (with the Wyckoff position 24d) in Table 1, is generally considered as the transition state between two first-nearest neighboring tetrahedral positions (but also between two octahedral positions, it is the same position). Nevertheless, $M$ is less stable than $o$ and $t$ sites. In the case of $\mathrm{H}, \mathrm{O}$ and $\mathrm{N}$ atoms, $M$ sites always have two imaginary frequencies, but for carbon, $M$ is a stable position.

For B atoms, results were similar to those obtained for oxygen: the stable positions are the octahedral sites, the ZPE is low and the $t$ 

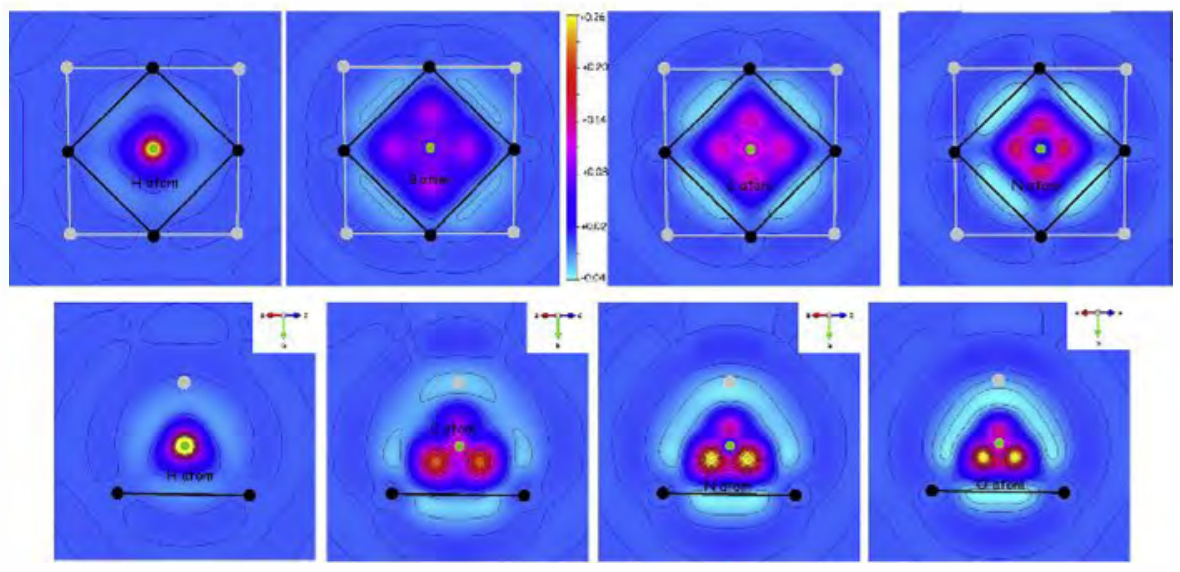

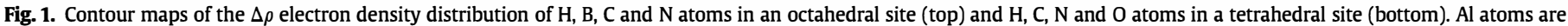
depicted in black in the plane and in gray in the upper plane. The green circle represents the position of $\mathrm{X}$ atom.

sites are unstable. We also tested the $M$ configuration for boron, without success: a calculation of the inter-atomic force interaction shows that this configuration has one imaginary frequency, $M$ is the transition state in the diffusion process between two octahedral sites, see David et al. [12].

Finally, we report the results on mono-vacancies $\left(V_{1}\right)$ and divacancies $\left(\mathrm{V}_{2}\right)$. In the case of $\mathrm{V}_{2}$, two configurations were considered: the first- and second-nearest neighboring positions, labeled $1 \mathrm{NN}$ and $2 \mathrm{NN}$, respectively. Our values (formation energies: $0.63,0.66$ and $0.62 \mathrm{eV}$ per vacancy, and volumes of formation $\Omega_{f}=$ $0.65,0.85$ and 0.88 u.a. per vacancy) are in agreement with previous studies $[9,10,26]$. $2 \mathrm{NN}$ is found slightly more stable than $1 \mathrm{NN}$, with a negative binding energy between vacancies, this is consistent with results obtained by Wang et al. [27].

In order to analyze the interactions between the interstitials and the metal, we analyzed the charge transfer using the Bader's charge as well as the differential charge density distributions, $\Delta \rho=\rho[A l+$ $X]-\rho[A l]-\rho[X]$. Results are summarized in Table 1 and plotted in Fig. 1, for octahedral and tetrahedral sites, only stable configurations are depicted. We used the same scale for all contour maps. We can see that all species acquire a part of the charge from the metal to partially fill their electronic shells: a positive charge transfer from the metal to the interstitial elements, from 1 to 3 electrons. In the case of $\mathrm{B}, \mathrm{C}, \mathrm{N}$ and $\mathrm{O}$ atoms, we clearly see that there is a charge transfer in the space between the solute and the first-nearest neighboring $\mathrm{Al}$ atoms, $\mathrm{Al}$ and $\mathrm{X}$ atoms form bonds. In the case of hydrogen, the charge transfer is different: it is mainly located around hydrogen. The electronic density-of-states (not depicted here) confirm these observations: $\mathrm{H}$ atoms, which have only $s$ shells, interact foremost with the states at the bottom of the valence levels of $\mathrm{Al}$ contrary to other elements, where their $p$ states interact with $\mathrm{Al}$ states. These results on the density-of-states are in agreement with those presented by Dai [28].

In the case of hydrogen, stability is partly controlled by the zeropoint energy, as already shown by Wolverton [25], which represents an important part of the solubility energy. However, in the case of other atoms, the ZPE is weak, so it is the competition between the elastic effects (measured through the volume of formation, elastic dipole, etc.) and the electronic interactions of the species with aluminum that seems to be responsible for the stability of the sites. It can be noted that there is a strong correlation between impurity electronegativity and site stability. The more the species is electronegative (as in the case of $\mathrm{N}$ and $\mathrm{O}$ atoms), the more it prefers to be in a tetrahedral site despite a strong elastic deformation.

To conclude on the general properties, we report the value of the elastic dipole $\left(\mathscr{P}_{i j}\right)$ induced by the solutes in their stable configurations and the volume of formation $\left(\Omega_{f}\right.$, in $\left.\AA^{3}\right)$. As explained by Clouet et al. [29], the elastic dipole is the adequate quantity to characterize the elastic distortion induced by the insertion of a defect in the network, according to the elastic theory. In the case of $o$ and $t$ sites, the elastic tensor has only one component in the diagonal. For the other cases (M sites and divacancies), the tensor $\mathscr{P}_{i j}$ contains several non-equal components and some off-diagonal elements, due to the symmetry break. All components are thus equal: $\mathscr{P}_{i j}=\delta_{i j} \mathscr{P}_{11}$. For $1 \mathrm{NN}$ and $2 \mathrm{NN}$ divacancies, we have two values, $\mathscr{P}_{11,22}$ and $\mathscr{P}_{33}$ and in the case of $M$, there are three components. To compute these elastic dipole components, we used the following expression:

$\mathscr{P}_{i j}=\sigma_{i j} / V$.

For different sizes of super-cells (up to 500 atoms), the volume was chosen equal to the equilibrium volume of the perfect structure (without defect) and afterwards only the atomic forces of the system with the impurity were relaxed. The strain $\sigma_{i j}$ is then measured after atomic relaxations (DFT values). For each element or vacancy,

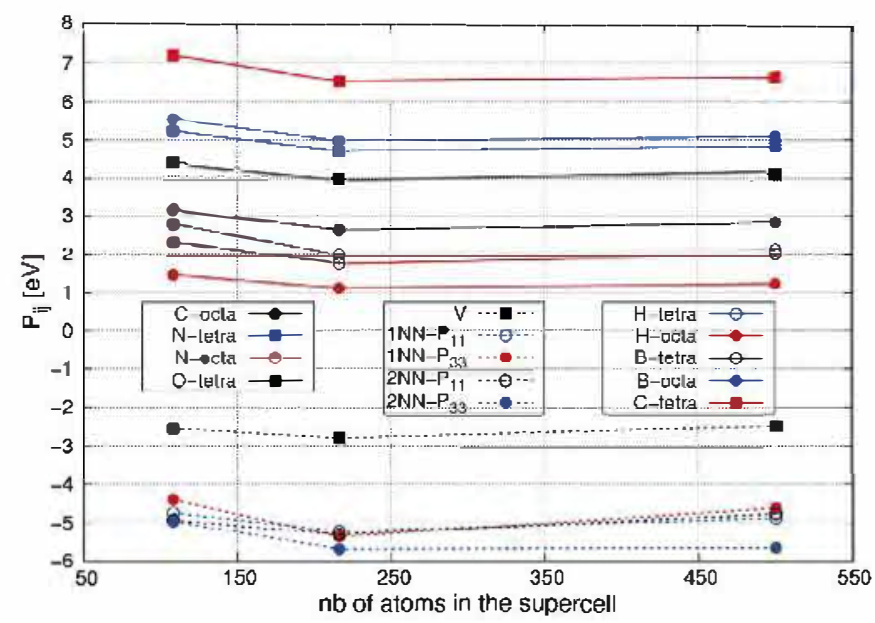

Fig. 2. Evolution of the elastic tensor $\mathscr{P}_{i j}$ (in eV) as a function of the size of the supercell. 
the evolution of the elastic tensor versus the size of the super-cell is depicted in Fig. 2 and the converged values are listed in Table 1.

Converged values are obtained for infinite box sizes. In our simulations, variations in $P_{i j}$ can be observed for small boxes. They are related to the interaction of impurities in adjacent cells. We note also that in order to have relatively well converged values, it is therefore necessary to use large simulation boxes ( $>200$ atoms). It can then be assumed that the values given in the table are converged to $0.2 \mathrm{eV}$.

The value of $\mathscr{P}_{11}$ is positive for all elements but negative in the case of the monovacancy. We also note that the evolution of $\mathscr{P}_{11}$ from $\mathrm{O}$ to $\mathrm{B}$ atoms is not monotonic, neither is volume of formation. In the case of $\mathrm{V}_{2}$, we note a small anisotropy: the value is twice as high as the monovacancy value, except for $\mathscr{P}_{33}\left[2 N N-V_{2}\right]$. We see that in octahedral sites $\mathscr{P}_{11}$ values are smaller than in tetrahedral sites. The stability of sites is a competition between elastic distortion of the lattice and electronic interactions (metal-X), this can be deduced by comparing elements.

In the light of these values, the lattice expansion, in the dilute limit, can be linked to the $\mathrm{X}$ concentration $C[X]$ by using the following expression (interstitial elements induce hydrostatic strain):

$a(C[X])=a_{0}(1+\delta \cdot C[X])$

with

$\delta=\frac{4}{a_{0}^{3}} \frac{\mathscr{P}_{11}}{3 B}=u \cdot \mathscr{P}_{11}$

where $B$ is the bulk modulus (approximately equal to $78 \mathrm{GPa}$ ) and $a_{o}$ the lattice parameter, equal to $4.04 \AA$. It gives, $u=0.041, \mathscr{P}_{11}$ is thus expressed in $\mathrm{eV}$. Insertion increases the lattice parameter, while in the case of vacancies, it decreases.

\section{Formation of $X_{m}$ clusters}

We hereinafter discuss the formation of clusters. We first consider the compact $X_{m}$ clusters in a perfect crystal. For $\mathrm{N}, \mathrm{H}$ and $\mathrm{O}$ ( $C$ and $B$ ) atoms, only tetrahedral (octahedral) positions were considered. DFT results are summarized in Table 2. In addition, initial configurations are displayed in Fig. 4.

We also considered an additional configuration for $\mathrm{X}_{4}$, in which $X$ atoms are located in $o$ sites and form a compact structure (case $c$ ): all $X$ atoms are then in the first-nearest neighbor positions ( $1 \mathrm{nn})$ with no $\mathrm{Al}$ atoms inside the pyramid formed by the $X_{4}$ cluster, contrary to the configuration (e), as represented in Fig. 4.

From a structural point of view, the relaxed positions are close to the ideal positions, interstitial atoms present small relaxations. Eq. (2) was used to compute formation energies $\left(H_{f}\left[X_{m}\right]\right)$ and Eq. (4) used for segregation energies $\left(E_{s e g}\left[X_{m}\right]\right)$. By analyzing segregation energies we were able to identify which aggregate is likely to be formed at the atomic scale: a negative value means that the $X_{m-1}$ cluster attracts one additional $X$ atom to form $X_{m}$ (see Fig. 3).

In the case of hydrogen, the ZPEs do not change significantly the energies (here, for simplification purposes only $\mathrm{H}$ atoms frequencies were computed). From $X_{2}$ configurations, we extracted $X$ $X$ pair interactions (labeled $\varepsilon_{X-X}^{n n}$ ): for the octahedral positions, (b) and (a) correspond to the first- and second-nearest neighboring configurations ( $1 \mathrm{nn}$ and $2 \mathrm{nn}$ ), respectively, and for the tetrahedral configurations, (c), (b) and (a) are the first-, second- and thirdnearest neighboring positions, respectively. Pair interactions can be used to build a generalized Ising model to study large clusters that cannot be obtained in DFT as illustrated in Refs. [3,30]. The pair interactions are thus directly equal to the segregation energies listed in Table 2 . We see that all species can form small $X_{m}$ clusters in aluminum $\left(E_{\text {seg }}<0\right)$. In the case of $\mathrm{H}, \mathrm{C}$ and $\mathrm{B}$ atoms, $\varepsilon_{X-X}^{1 n n}$ is negative: it is equal to $-0.06,-0.30$ and $-0.13 \mathrm{eV}$, respectively. The energy of the other (pair interaction) components can be considered equal to zero. In the case of $\mathrm{N}$ atoms, we obtain a positive value for $\varepsilon_{X-X}^{1 n n}$ (atoms repel), but a high negative value for $\varepsilon_{X-X}^{2 n n}(-0.26 \mathrm{eV})$.

Table 2

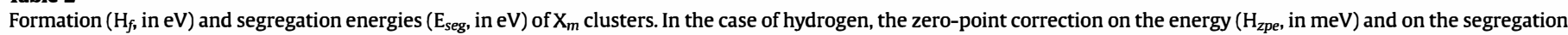

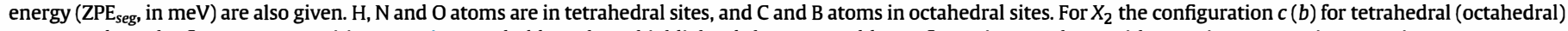
corresponds to the first-nearest position, see Fig. 5. In bold, we have highlighted the most stable configurations or those with negative segregation energies.

\begin{tabular}{|c|c|c|c|c|c|c|c|c|c|c|c|c|c|}
\hline \multicolumn{2}{|c|}{ config } & \multicolumn{2}{|l|}{ nitrogen } & \multicolumn{4}{|c|}{ hydrogen } & \multicolumn{2}{|l|}{ oxygen } & \multicolumn{2}{|l|}{ carbon } & \multicolumn{2}{|l|}{ boron } \\
\hline & & $\mathrm{H}_{f}$ & $E_{s e g}$ & $\mathrm{H}_{f}$ & $\mathrm{H}_{z p e}$ & $E_{\text {seg }}$ & $\mathrm{ZPE}_{\text {seg }}$ & $\mathrm{H}_{f}$ & $\mathrm{E}_{\text {seg }}$ & $\mathrm{H}_{f}$ & $\mathrm{E}_{\text {seg }}$ & $\mathrm{H}_{f}$ & $\mathrm{E}_{\text {seg }}$ \\
\hline \multirow[t]{2}{*}{$X_{1}$} & octa & -0.056 & - & 0.823 & -68 & - & - & unstable & - & 1.322 & - & 1.229 & - \\
\hline & tetra & -0.904 & - & 0.717 & 13 & - & - & -3.478 & - & 1.691 & - & unstable & - \\
\hline \multirow[t]{3}{*}{$X_{2}$} & a & $-1.715^{3}$ & 0.093 & $1.450^{3}$ & 36 & 0.017 & 11 & $-6.862^{3}$ & 0.094 & $2.643^{2}$ & -0.001 & $2.535^{2}$ & 0.078 \\
\hline & b & $-2.065^{2}$ & -0.258 & $1.484^{2}$ & 20 & 0.051 & -5 & $-7.193^{2}$ & -0.237 & $2.337^{1}$ & -0.307 & $2.319^{1}$ & -0.138 \\
\hline & c & $-1.434^{1}$ & 0.373 & $1.366^{1}$ & 23 & -0.067 & -3 & $-7.008^{1}$ & -0.052 & - & - & - & - \\
\hline \multirow[t]{3}{*}{$X_{3}$} & a & -2.296 & 0.673 & 2.028 & -97 & -0.055 & -132 & -10.730 & -0.059 & 3.339 & -0.319 & 3.420 & -0.128 \\
\hline & b & -2.619 & 0.350 & 2.140 & -98 & 0.058 & -133 & -10.717 & -0.049 & 3.030 & -0.629 & 3.238 & -0.309 \\
\hline & c & -3.461 & -0.492 & 2.247 & -115 & 0.164 & -151 & -11.124 & -0.454 & - & - & - & - \\
\hline \multirow[t]{6}{*}{$X_{4}$} & a & -2.243 & 2.122 & 2.598 & 45 & -0.147 & 130 & -14.333 & 0.270 & 4.035 & -0.316 & 4.366 & -0.101 \\
\hline & b & -3.737 & 0.628 & 2.862 & 16 & 0.117 & 100 & -14.810 & -0.207 & 3.749 & -0.603 & 4.161 & -0.306 \\
\hline & c & -4.136 & 0.229 & 2.721 & 32 & -0.023 & 116 & -14.622 & -0.019 & $3.445^{4}$ & -0.906 & $3.957^{4}$ & -0.510 \\
\hline & d & -3.476 & 0.889 & 2.861 & 61 & 0.117 & 145 & -14.478 & 0.124 & - & - & - & - \\
\hline & e & -5.090 & -0.725 & 3.111 & 8 & 0.367 & 92 & -14.649 & -0.046 & - & - & - & - \\
\hline & f & -3.198 & 1.167 & 2.720 & 53 & -0.024 & 137 & -14.422 & 0.180 & - & - & - & - \\
\hline \multirow[t]{3}{*}{$X_{5}$} & a & -4.529 & 1.465 & 3.318 & 46 & 0.003 & -13 & -18.311 & -0.023 & 4.202 & -0.868 & 5.007 & -0.382 \\
\hline & b & -4.169 & 1.824 & 3.468 & 57 & 0.154 & -2 & -18.615 & -0.327 & - & - & - & - \\
\hline & c & -5.838 & 0.160 & 3.553 & 37 & 0.238 & -22 & -18.714 & -0.426 & - & - & - & - \\
\hline \multirow[t]{3}{*}{$\mathrm{X}_{6}$} & $\mathrm{a}$ & -6.540 & 0.198 & 4.038 & 63 & 0.004 & 4 & -22.657 & -0.465 & 4.432 & -1.092 & 5.826 & -0.410 \\
\hline & b & -4.338 & 2.401 & 3.945 & 67 & -0.089 & 8 & -22.186 & 0.006 & - & - & - & - \\
\hline & c & -4.665 & 2.072 & 4.120 & 90 & 0.086 & 31 & -22.871 & -0.680 & - & - & - & - \\
\hline \multicolumn{2}{|l|}{$X_{7}$} & -7.236 & 0.207 & 4.582 & 109 & -0.079 & 29 & -26.261 & 0.088 & - & - & - & - \\
\hline \multicolumn{2}{|l|}{$X_{8}$} & -2.342 & 5.798 & 5.203 & 138 & -0.096 & 16 & -29.446 & 0.294 & - & - & - & - \\
\hline
\end{tabular}

$11 \mathrm{nn},{ }^{2} 2 \mathrm{nn}$ and ${ }^{3} 3 \mathrm{nn}$ configurations; ${ }^{4}$ corresponds to the compact $\mathrm{X}_{4}$ structure. 

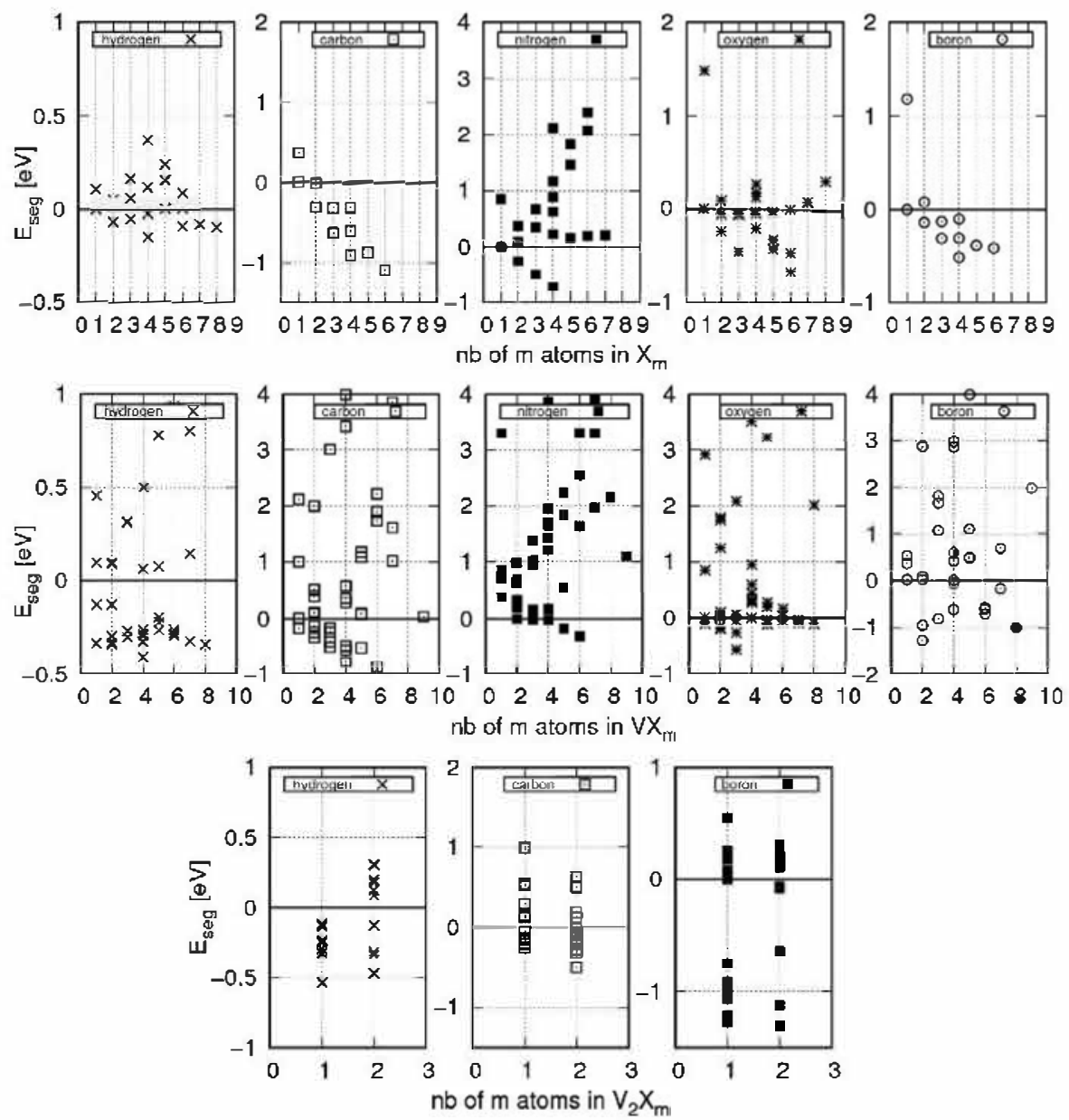

Fig. 3. Segregation energies of $X_{m}, V X_{m}$ and $V_{2} X_{m}$ (from top to bottom) clusters as a function of the number $m$ of $X$ atoms.
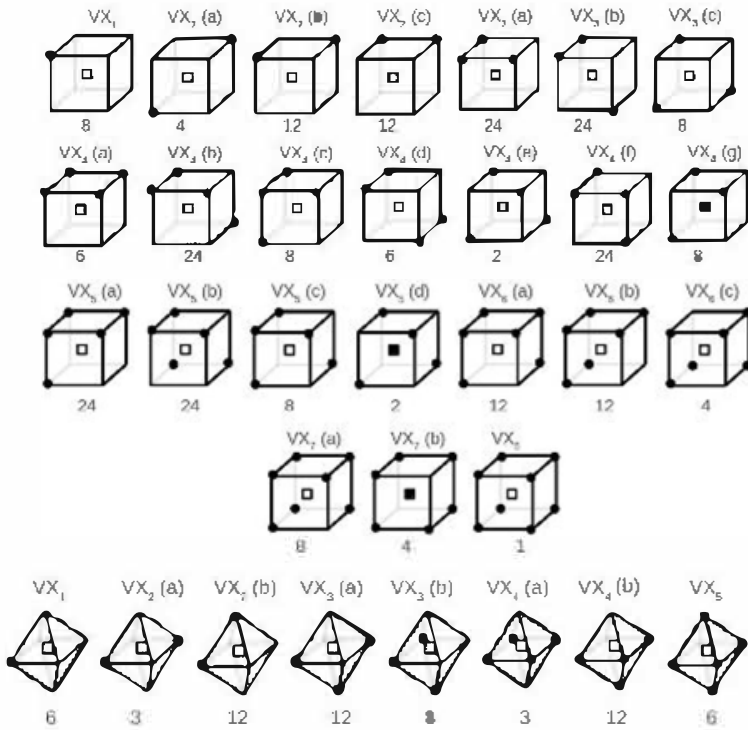

$\mathrm{Vx}_{4}(\mathrm{~b})$

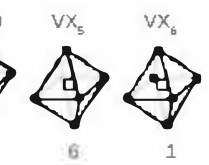

Fig. 4. : Schematic representation of $V X_{m}$ configurations with their degeneracy. Only $X$ atoms (black balls) and the vacancy (square) are represented. In the case of $X_{m}$ clusters, we used the same configurations where the vacancy is filled by an $\mathrm{Al}$ atom. On top (bottom), we represent tetrahedral (octahedral) configurations formings a cubic (bipyramidal) structure around the vacancy.
For 0 atoms, $\varepsilon_{X-X}^{1 n n}$ and $\varepsilon_{X-X}^{2 n n}$ are both negative. Apart from oxygen (especially for large clusters), these pair interactions can reproduce $\mathrm{X}_{m}$ cluster energies without additional DFT calculations. These parameters are sufficient to reproduce $X_{m}$ energies with a great accuracy.

One notes that $\mathrm{H}, \mathrm{C}$ and $\mathrm{B}$ atoms can form large compact aggregates. The more $m$ increases, the more stable $C_{m}$ clusters are, and they are organized in a NaCl-like structure (see Appendix A.2). In the case of nitrogen, compact clusters are not preferred, but a structure emerges from DFT energies and pair energies. In this structure, each $\mathrm{N}$ atom is located in the second-nearest position of another $\mathrm{N}$ atom in the fcc lattice of $\mathrm{Al}$. It corresponds to a zincblende structure. Experimentally, as explained in the introduction, there is only one intermediate alloy, the AlN structure. The difference in energy between both phases (zincblende and wurtzite) is low, but tends to favor wurtzite (see Appendix A.2). One can expect that for "high" concentrations, the zincblende structure transforms into the wurtzite phase.

In the case of $\mathrm{O}$ atoms, it is difficult to simply extract a general trend. Clusters are energetically stable (up to 60 atoms in tetrahedral positions), but larger configurations are not favored. The pair interactions can not explain these results. However, as we will see below, such clusters should not exist from a thermodynamic point of view. 


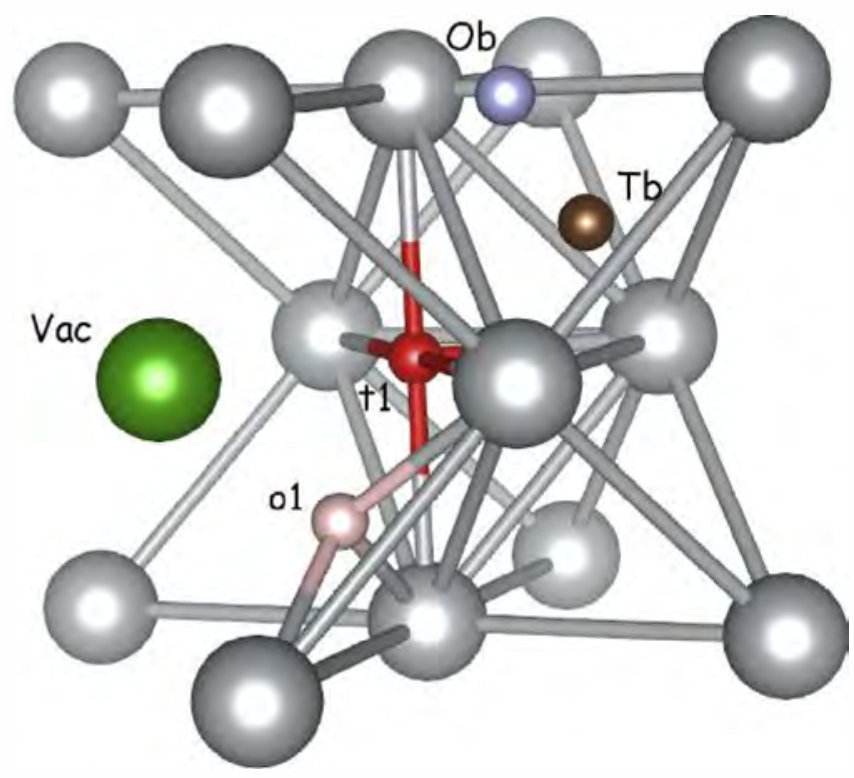

Fig. 5. $o_{1}$ and $t_{1}$ sites position in the cluster and $o_{b}$ and $T_{b}$ sites in the bulk.

\section{Formation of $V X_{m}$ clusters}

We now investigate the interactions between a vacancy and one or several interstitial elements. As stated in previous works [1-4,7,31], we will see that some of them $(\mathrm{H}, \mathrm{O}, \mathrm{B}$ and $\mathrm{C})$ interact with vacancies and can form $\mathrm{VX}_{m}$ clusters.

In previous works $[5,9,10]$, aluminum has been used several times as reference system in the study of $\mathrm{H}$ clusters formation, but no studies have been carried out on other interstitial elements (C, $\mathrm{N}, \mathrm{O}$ and $\mathrm{B}$ ). Different positions were checked: either in the octahedral sites (labeled $o_{1}$ ) or in the tetrahedral $\left(t_{1}\right)$ sites inside the first-nearest coordination sphere of the vacancy (see Fig. 5).

To look for intermediate positions, especially for low $m$ values, we moved atoms from their ideal positions $\left(o_{1}\right.$ and $\left.t_{1}\right)$ and towards the vacancy. We obtain only two stable positions: $o_{1}$ and $t_{1}$, near the ideal position. Table 3 lists the formation and segregation energies of clusters. Results are depicted in Fig. 3.

As seen above, the substituted positions are energetically not preferred and unstable for all species studied, the vacancy is then restored and a vacancy-X pair is built.

In the case of $\mathrm{N}$ atoms, we find that for low $\mathrm{N}$ contents $\mathrm{VN}_{i}$ segregation energies are always positive: for both octahedral and tetrahedral sites and for any alternative position, contrary to what is observed in iron [3,32]. Nitrogen atoms and vacancies repel each other, thus preventing the formation of clusters. However, some clusters show negative segregation energies: $\mathrm{VN}_{4}, \mathrm{VN}_{5}$ and $\mathrm{VN}_{6}$,

Table 3

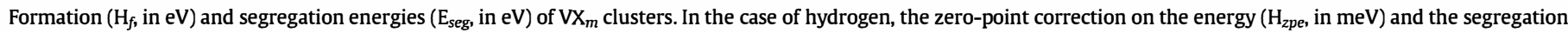

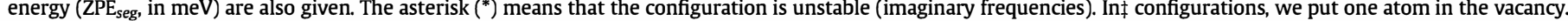
Some configurations converge through the same cluster after relaxation, they are labeled "1", "2" and "3".

\begin{tabular}{|c|c|c|c|c|c|c|c|c|c|c|c|c|c|c|}
\hline \multirow[t]{2}{*}{ config } & & & \multicolumn{2}{|l|}{ nitrogen } & \multicolumn{4}{|c|}{ hydrogen } & \multicolumn{2}{|l|}{ oxygen } & \multicolumn{2}{|l|}{ carbon } & \multicolumn{2}{|l|}{ boron } \\
\hline & & & $\mathrm{H}_{f}$ & $\mathrm{E}_{\text {seg }}$ & $\mathrm{H}_{f}$ & $\mathrm{H}_{z p e}$ & $\mathrm{E}_{\text {seg }}$ & $\mathrm{ZPE}_{\text {seg }}$ & $\mathrm{H}_{f}$ & $\mathrm{E}_{\text {seg }}$ & $\mathrm{H}_{f}$ & $\mathrm{E}_{\text {seg }}$ & $\mathrm{H}_{f}$ & $\mathrm{E}_{\text {seg }}$ \\
\hline \multirow[t]{2}{*}{$X_{1}$} & octa & & -0.056 & - & 0.823 & -68 & - & - & unstable & - & 1.322 & - & 1.229 & - \\
\hline & tetra & & -0.904 & - & 0.717 & 13 & - & - & -3.478 & - & 1.691 & - & unstable & - \\
\hline \multirow[t]{3}{*}{$\mathrm{VX}_{1}$} & sub & & $3.040^{*}$ & 3.308 & $1.806^{*}$ & -37 & 0.454 & -50 & $0.066^{*}$ & 2.908 & $4.075^{*}$ & 2.118 & $2.399^{*}$ & 0.535 \\
\hline & octa & & 0.102 & 0.371 & 1.225 & -105 & -0.127 & -118 & -1.989 & 0.853 & 1.792 & -0.165 & 1.889 & 0.025 \\
\hline & tetra & & 0.435 & 0.704 & 1.017 & 10 & -0.335 & -2 & -2.926 & -0.084 & 2.959 & 1.001 & 2.226 & 0.362 \\
\hline \multirow[t]{5}{*}{$\mathrm{VX}_{2}$} & octa & $a$ & -0.479 & 0.323 & 1.824 & $-208^{*}$ & 0.090 & -232 & -4.655 & 1.750 & 2.875 & -0.238 & 3.136 & 0.013 \\
\hline & & b & -0.612 & 0.189 & 1.828 & $-173^{*}$ & 0.095 & -196 & -5.155 & 1.250 & 2.779 & -0.334 & 3.201 & 0.079 \\
\hline & tetra & $a$ & 0.176 & 0.977 & 1.402 & 16 & -0.332 & -7 & -6.561 & -0.156 & 3.517 & 0.399 & 5.990 & 2.867 \\
\hline & & b & 0.187 & 0.988 & 1.393 & 18 & -0.340 & -5 & -6.578 & -0.173 & 3.217 & 0.097 & 2.155 & -0.967 \\
\hline & & c & -0.115 & 0.686 & 1.437 & 35 & -0.297 & 12 & -6.368 & 0.037 & 2.996 & -0.122 & 1.846 & -1.276 \\
\hline \multirow[t]{5}{*}{$\mathrm{VX}_{3}$} & octa & $\mathrm{a}$ & -1.354 & 0.162 & 2.424 & $-198^{*}$ & 0.314 & -228 & -7.971 & 2.085 & 3.698 & -0.403 & 4.735 & 1.661 \\
\hline & & b & -1.524 & -0.008 & 2.429 & -238 & 0.319 & -268 & unstable & & 3.598 & -0.503 & 4.886 & 1.811 \\
\hline & tetra & $a$ & -0.498 & 1.018 & 1.841 & 59 & -0.269 & 28 & -9.991 & 0.065 & 3.945 & -0.162 & 2.252 & -0.822 \\
\hline & & b & -0.582 & 0.934 & 1.806 & 43 & -0.303 & 12 & -10.105 & -0.049 & 3.880 & -0.232 & 4.152 & 1.078 \\
\hline & & c & -0.135 & 1.381 & 1.760 & 52 & -0.350 & 20 & -10.299 & -0.243 & 5.184 & 1.083 & 2.783 & -0.291 \\
\hline \multirow[t]{9}{*}{$\mathrm{VX}_{4}$} & octa & a & -2.252 & 0.175 & 3.025 & -298 & 0.502 & -353 & -10.559 & 3.516 & 4.439 & -0.480 & 6.339 & 2.858 \\
\hline & & b & -2.443 & -0.015 & 2.587 & $-85^{*}$ & 0.064 & -140 & -13.121 & 0.953 & 4.368 & -0.552 & 6.457 & 2.976 \\
\hline & tetra & $a$ & -0.772 & 1.656 & 2.260 & 106 & -0.263 & 51 & -13.475 & 0.600 & 5.280 & 0.360 & $2.855^{3}$ & -0.626 \\
\hline & & b & -1.223 & 1.205 & 2.196 & 65 & -0.327 & 10 & -13.787 & 0.288 & 5.222 & 0.289 & $2.855^{3}$ & -0.626 \\
\hline & & c & -0.723 & 1.705 & 2.230 & 81 & -0.293 & 25 & -13.756 & 0.319 & 4.159 & $-0.769^{2}$ & 3.486 & 0.005 \\
\hline & & d & -1.008 & 1.420 & 2.199 & 65 & -0.325 & 9 & -13.743 & 0.332 & 5.532 & 0.574 & 4.063 & 0.582 \\
\hline & & e & -0.480 & 1.948 & 2.114 & 40 & -0.409 & -16 & -14.079 & $-0.004^{1}$ & 8.353 & 3.433 & 3.882 & 0.401 \\
\hline & & f & -0.987 & 1.441 & 2.237 & 81 & -0.286 & 25 & -13.627 & 0.448 & 5.493 & 0.574 & 3.416 & -0.065 \\
\hline & & $g \ddagger$ & 1.446 & 3.873 & 2.403 & $-100^{*}$ & 0.076 & -145 & -14.079 & $-0.004^{1}$ & 4.159 & $-0.769^{2}$ & 3.448 & -0.033 \\
\hline \multirow[t]{5}{*}{$\mathrm{VX}_{5}$} & octa & & -3.515 & -0.168 & 3.608 & $-308^{*}$ & 0.777 & -360 & -14.331 & 3.226 & 4.958 & -0.514 & 8.083 & 3.010 \\
\hline & tetra & $a$ & -1.512 & 1.835 & 2.636 & 123 & -0.195 & 71 & -17.266 & 0.291 & 6.558 & 1.085 & 5.192 & 0.118 \\
\hline & & b & -2.812 & 0.535 & 2.611 & 103 & -0.220 & 51 & -17.357 & 0.199 & 6.671 & 1.171 & 4.578 & -0.495 \\
\hline & & c & -1.105 & 2.242 & 2.569 & 84 & -0.262 & 32 & -17.592 & -0.035 & 5.567 & 0.095 & 4.558 & -0.516 \\
\hline & & $\mathrm{d} \ddagger$ & 1.648 & 4.995 & 2.907 & - & 0.076 & - & unstable & & 5.567 & 0.095 & 4.558 & -0.516 \\
\hline \multirow[t]{4}{*}{$\mathrm{VX}_{6}$} & octa & & -4.726 & -0.307 & 4.221 & -416 & 0.936 & -513 & -16.830 & 4.239 & 5.415 & -0.865 & 9.808 & 2.886 \\
\hline & tetra & $\mathrm{a}$ & -2.780 & 1.639 & 2.992 & 141 & -0.293 & 44 & -21.125 & -0.056 & 8.179 & 1.899 & 5.071 & -1.852 \\
\hline & & b & -1.870 & 2.548 & 3.021 & 159 & -0.264 & 62 & -20.892 & 0.177 & 8.497 & 2.216 & 5.172 & -1.750 \\
\hline & & $c \ddagger$ & -1.104 & 3.315 & 3.004 & 139 & -0.281 & 42 & -21.033 & 0.037 & 8.021 & 1.741 & 5.200 & -1.722 \\
\hline \multirow[t]{3}{*}{$\mathrm{VX}_{7}$} & octa & & -3.664 & 1.965 & 4.509 & - & 0.800 & - & unstable & & 7.756 & 1.019 & unstable & \\
\hline & tetra & $\mathrm{a}$ & -2.320 & 3.309 & 3.386 & 193 & -0.323 & 39 & -24.676 & -0.073 & 10.599 & 3.862 & 6.124 & -0.175 \\
\hline & & bł & -1.724 & 3.905 & 3.854 & - & 0.145 & - & unstable & & 10.464 & 3.727 & 6.985 & 0.686 \\
\hline $\mathrm{VX}_{8}$ & tetra & & -1.074 & 2.151 & 3.762 & 237 & -0.341 & 31 & -28.244 & -0.090 & 13.936 & 2.015 & 6.343 & -1.009 \\
\hline
\end{tabular}


where $\mathrm{N}$ atoms are in $o_{1}$. As we will see below, these configurations are not formed.

In the case of $\mathrm{H}$ atoms, we reproduce the segregation energies of clusters obtained in earlier works [8-10] on $\mathrm{VH}_{m}$ clusters. The most stable configurations inside the vacancy are the $t_{1}$ positions, as in the bulk. The segregation energies are almost constant, approximately equal to $-0.300 \mathrm{eV}$, regardless the number of hydrogen atoms inside the cluster (see Fig. 3). The zero-point energy of $\mathrm{H}$ atoms, computed for all configurations, slightly modifies the segregation energies: from 10 to $100 \mathrm{meV}$, see Table 3 . These simulations also enabled to show that some configurations are unstable (they have almost three imaginary frequencies), especially some $\mathrm{VH}_{i}$ clusters, with $\mathrm{H}$ atoms located in $\mathrm{o}_{1}$ sites (configurations labeled by an asterisk ( $\left.{ }^{*}\right)$ in the table). In addition, we tested configurations proposed by Lu et al. [9]. This was unsuccessful: $\mathrm{H}_{2}$ molecules are less stable than dissociated elements inside the vacancy.

Contrary to nickel [7] where the binding energy between oxygen and vacancy is strong, in aluminum, there is a small attractive energy $\left(\varepsilon_{V-N}^{1 n n} \simeq-0.084 \mathrm{eV}\right)$. From 1 to 3 oxygen in a vacancy, the segregation energy is slightly negative. We also observed that 0 atoms prefer to stay in a quasi-tetrahedral configuration. In some $\mathrm{O}_{2}$ cases (for $\mathrm{VO}_{4}$ and $\mathrm{VO}_{3}$ ), $\mathrm{O}$ atoms move away from the vacancy. This last result is in agreement with our previous results showing that 0 sites are unstable for $\mathrm{O}$ atoms.

In the case of $C$ atoms, the interaction with a vacancy energetically favors segregation. $C$ atoms prefer to occupy the octahedral position $o_{1}$, the pair interaction is equal to $\varepsilon_{V-C}^{1 n n} \simeq-0.165 \mathrm{eV}$. The detailed analysis of relaxed configurations shows that in some cases (configurations labeled $\ddagger$ in Table 3 ), when $C$ atoms are in $t_{1}$ sites, new stable clusters are formed: in such configurations one $C$ atom located in a $t_{1}$ site moves inside the vacancy. In such configurations, a diamond like structure (Fig. $4, \mathrm{VX}_{4}, \mathrm{~d}$ configuration), the central atom forms bounds with other carbons. The same configurations were also studied for others elements, but results were inconclusive. DFT simulations indicate that $\mathrm{VC}_{6}$ clusters could be formed.

Concerning the interactions between vacancies and $\mathrm{B}$ atoms, the situation is different. As for $\mathrm{N}$ atoms, small clusters $\left(\mathrm{VB}_{1}\right)$ are energetically unfavorable, but some configurations have strong negative segregation energies, especially $\mathrm{VB}_{2}, \mathrm{VB}_{3}$ and $\mathrm{VB}_{4}$. Configurations with $\mathrm{B}$ atoms in $o_{1}$ positions are thermodynamically unfavorable. The most stable configurations are those where boron atoms are put in $t_{1}$ sites initially. However, contrary to other species where relaxed configurations are close to ideal positions, B atoms move greatly in the clusters. The reorganization around the vacancy is relatively high. Simple pair-interaction energies are thus inefficient to reproduce the interactions inside the vacancy.

Finally, as for $X_{m}$ clusters, we extracted the pair-interaction energies $\varepsilon_{V-X}^{1 n n}$ from VX clusters. For $N$ atoms the value is always positive, and for $\mathrm{H}, \mathrm{C}$ and $\mathrm{O}$ we obtain $-0.33,-0.16$ and $-0.08 \mathrm{eV}$, respectively. The lowest pair-interaction is thus obtained for $\mathrm{H}$ atoms. As seen above, in the case of $B$ atoms, a simple pairinteraction model is not accurate enough to capture the physics. The characteristics of large clusters can not be deduced from a simple model.

\section{6. $V_{2} X_{m}$ clusters}

Finally, we report results on $\mathrm{V}_{2} \mathrm{X}_{m}$ clusters. We explored the segregation of species in both divacancy configurations: $1 \mathrm{NN}$ and 2NN.

We studied octahedral and tetrahedral configurations but also "substituted" sites ( $d$ cases), corresponding to one of the two vacancies being occupied by an element. We also considered

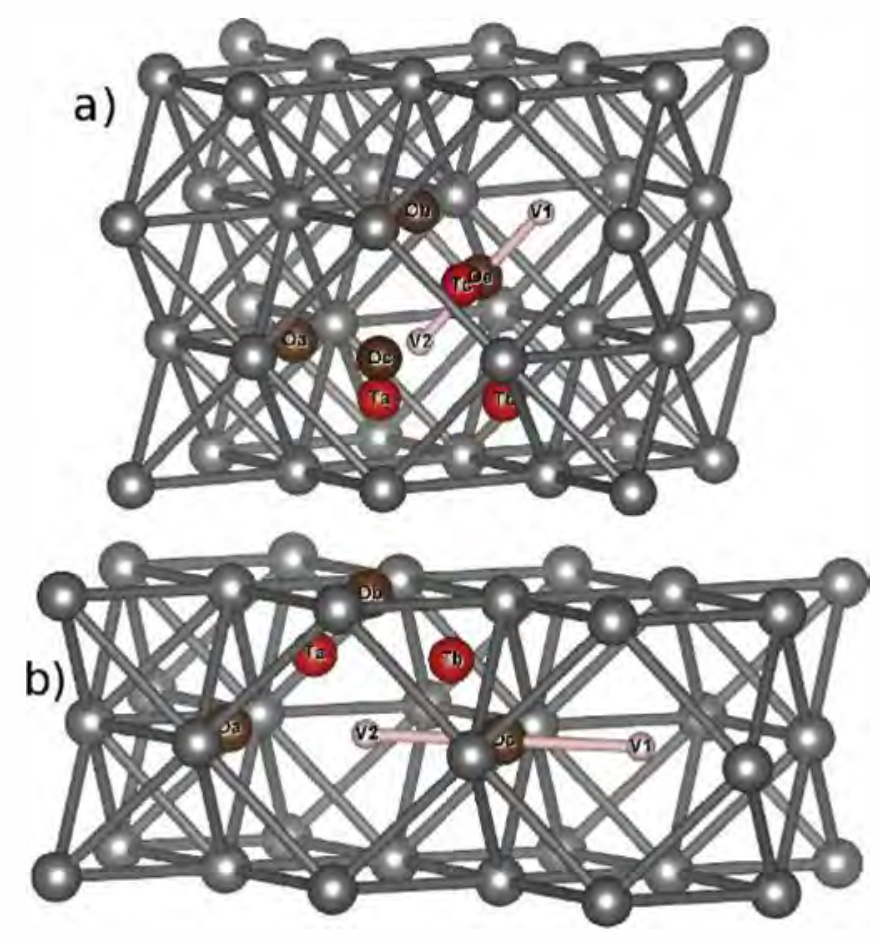

Fig. 6. Schematic representation of $V_{2} X_{m}$ configurations: tetrahedral sites in read and the octahedral sites in brown. The Od case corresponds to the "substitution" of one vacancy (V2, for example).

configurations where $X$ atoms are between the divacancies ( $e$ and $c$ for $1 \mathrm{NN}$ and $2 \mathrm{NN}$, respectively). Configurations are displayed in Fig. 6. We inserted two segregation energies: the first one $\left(E_{\text {seg }}^{1}\right)$ corresponds to the energy gain (loss) when adding one vacancy in VX (we considered the most stable VX configuration as reference state) and the second one $\left(E_{\text {seg }}^{2}\right)$ corresponds to the energy gain (loss) when adding an $X$ atom in $\mathrm{V}_{2}$. Table 4 summarizes these results.

Similarly to VX clusters, $\mathrm{N}$ and $\mathrm{O}$ atoms segregate poorly in $\mathrm{V}_{2}$ divacancies. The segregation energy is, at best, slightly negative. We can expect that these elements will not form $V_{2} X_{m}$ clusters. On the contrary, there is an attractive interaction between $\mathrm{H}$ and $V_{2}$, equivalent to what is obtained for the monovacancy $\left(E_{\text {seg }}^{2} \simeq-300\right.$ $\mathrm{meV}$ ), and for $\mathrm{C}$ atoms some octahedral configurations remain stable. In the case of B atoms, $V_{2} B_{m}$ are energetically less stable than $V B_{m}$.

Some additional $V_{2} X_{m}$ configurations were explored, where $\mathrm{m}=2$ and 3, see Fig. 3. For $\mathrm{H}$ atoms the segregation is favorable, while for $B$ and $C$ atoms, the energy gain is low $\left(E_{\text {seg }}^{2}<0\right)$. We inserted these modified results into the statistical model but, since the melting point of $\mathrm{Al}$ is relatively low, these clusters will not appear in significant concentration.

\section{Statistical approach}

We now discuss the variation in defect concentrations as a function of $\mathrm{X}$ atom concentration and temperature by using the statistical approach described in part 2 and the formation energies given above. For simplification purposes, we did not present the results as a function of the chemical potential, $\mu[X]$. Indeed, for a given value of $\mathrm{T}$, each value of $\mu[X]$ gives one value of total $X$ concentration, $C_{\text {tot }}[X]$. We can thus plot independently the different results either as a function of $C_{\text {tot }}[X]$ or as a function of $\mu[X]$. The final 
Table 4

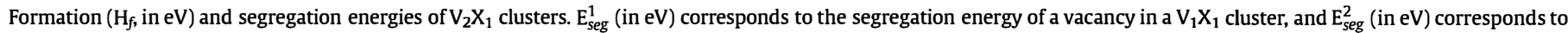
the segregation energy of $\mathrm{X}$ in a $\mathrm{V}_{2}$ divacancy. " $\mathrm{Od}=\mathrm{V} 2$ " means that one $\mathrm{X}$ atom occupies the vacancy.

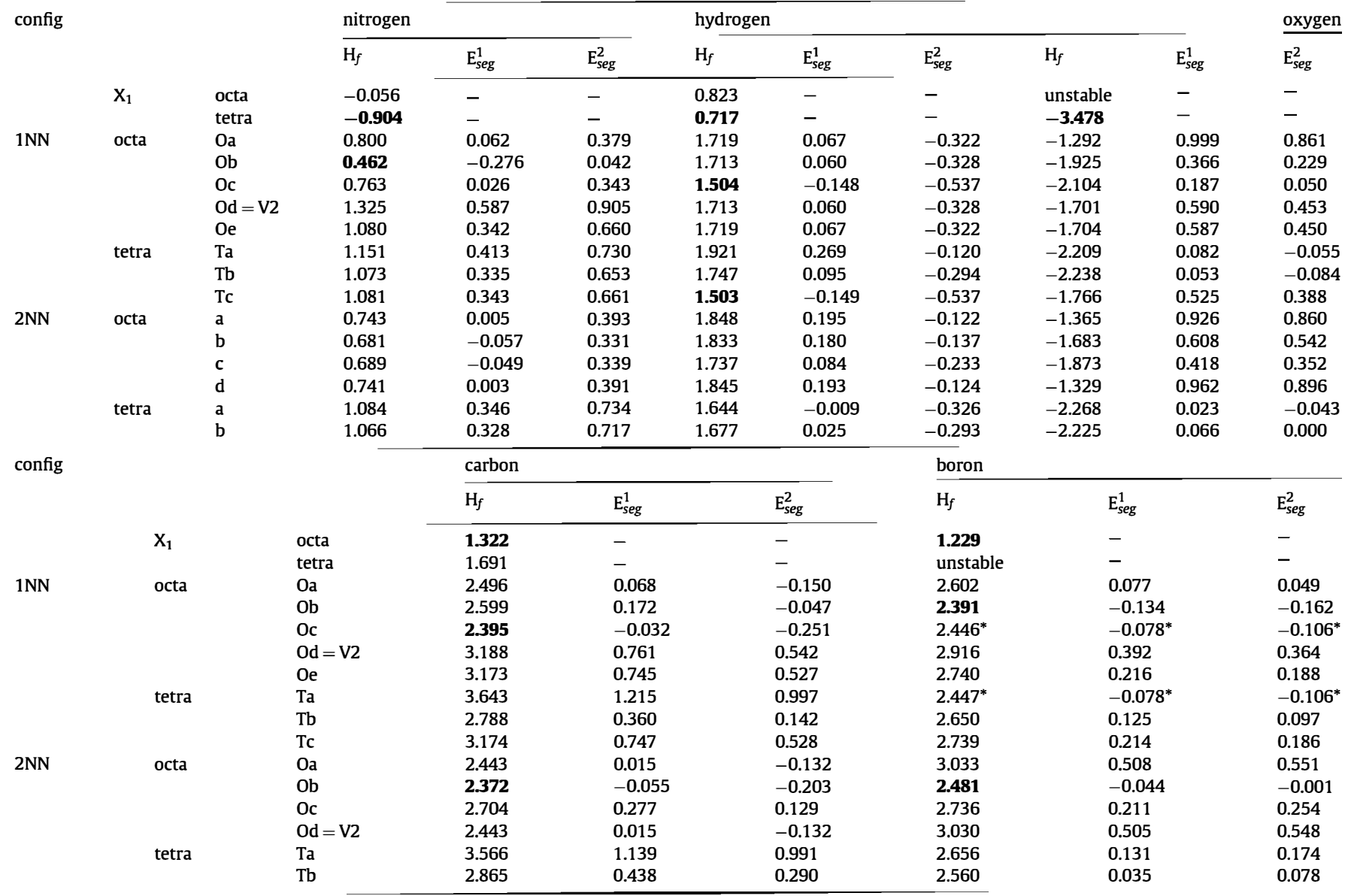
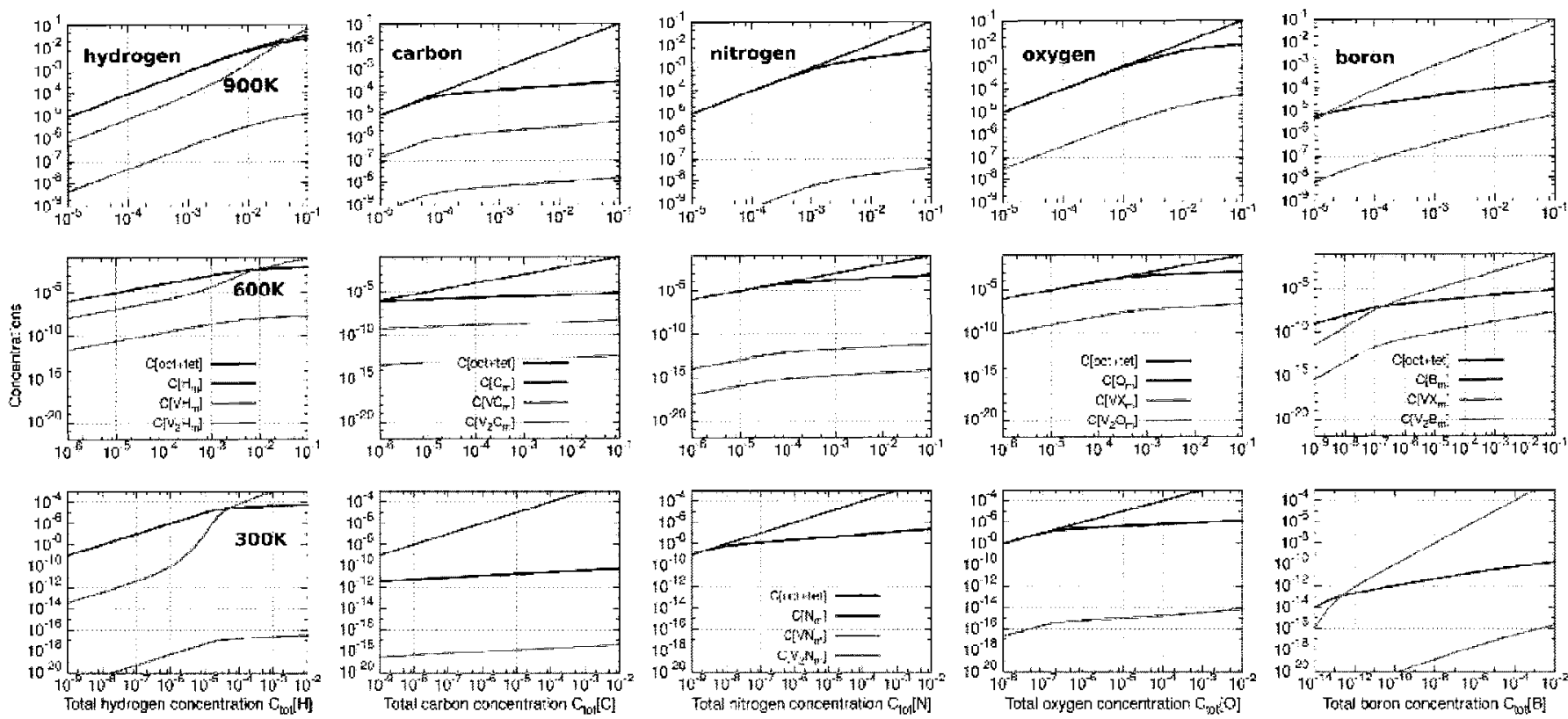

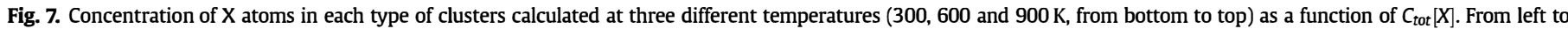

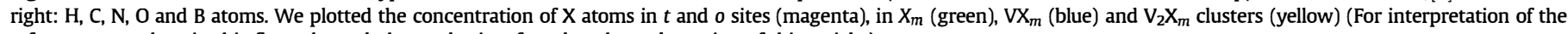
references to colour in this figure legend, the reader is referred to the web version of this article.). 

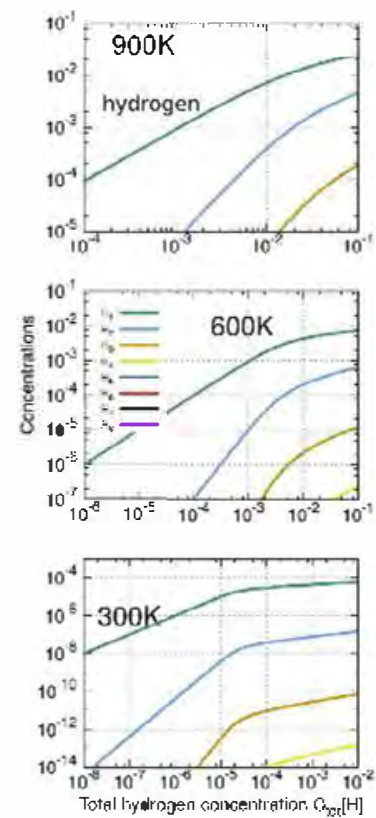
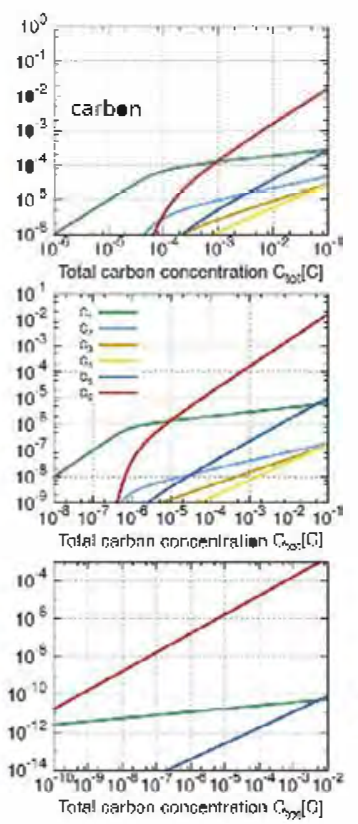
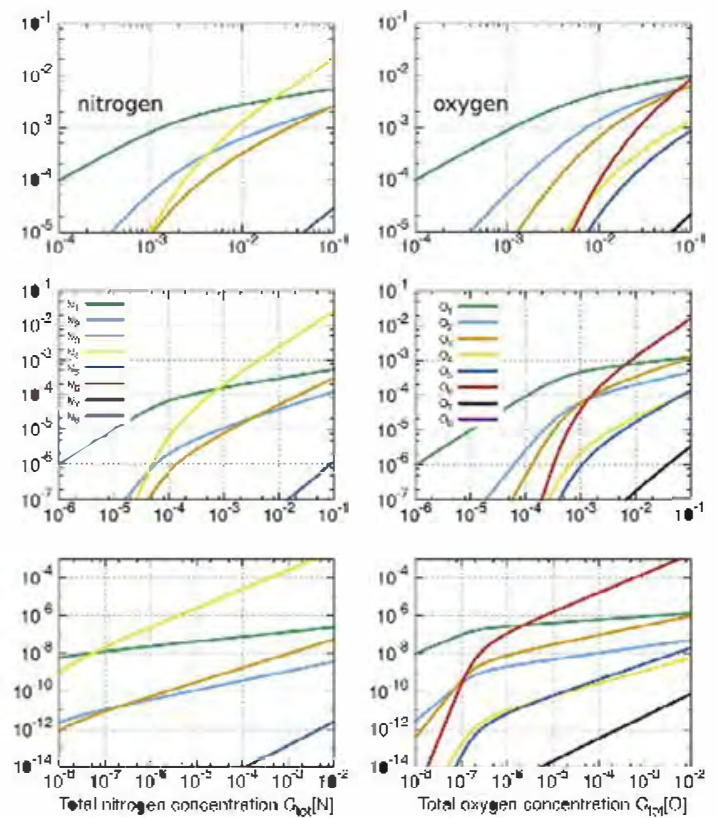
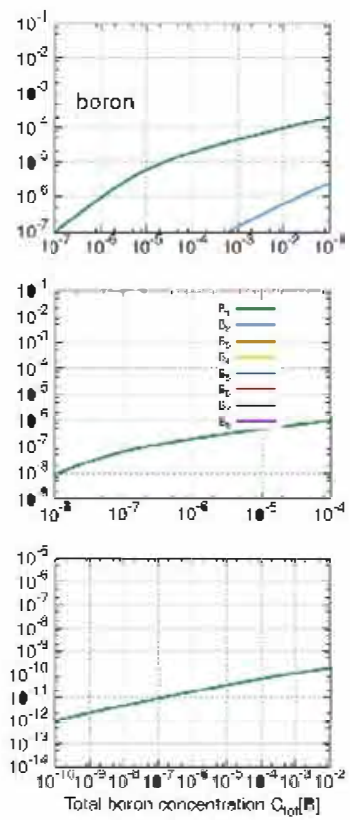

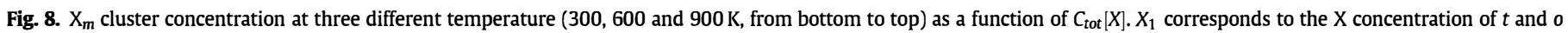
sites together. From left to right: H,C, N, O and B atoms.

results are depicted in Figs. 7 and 8.

Figs. 9 and 10 detail $\mathrm{VX}_{m}$ concentrations for $\mathrm{H}$ and $\mathrm{B}$ atoms. In the case of other species ( $\mathrm{C}, \mathrm{N}$ and $\mathrm{O}$ atoms), $\mathrm{VX} m$ concentrations are considerably lower than the free vacancies, they are therefore not shown for simplification purposes.

Results, plotted in Fig. 7 enable to predict the form under which $\mathrm{X}$ atoms should be when located in aluminum: either alone (in octahedral or tetrahedral sites) or in clusters. For experimental concentrations (10-100 appm), $\mathrm{O}, \mathrm{N}$ and $\mathrm{C}$ atoms are mainly located in $X_{m}$ clusters at low T (green curves). The fraction of free atoms (magenta curves) corresponding to these three species is thus negligible. At high temperature (close to the melting point, i.e. $900 \mathrm{~K}$ ), due to thermal effects, $X_{m}$ are broken, species are free. To find $X_{m}$ clusters at high T, very high concentrations of $X$ would be required, beyond what is observed experimentally. Whichever the case, all three species ( $\mathrm{C}, \mathrm{N}$ and $\mathrm{O}$ ) do not form clusters with vacancies, even at low temperature. The only clusters formed are $\mathrm{X}_{m}$ clusters. The vacancy concentration remains therefore unchanged, and is therefore fixed by thermal vacancies. On the other hand, the effect on vacancy concentration of the presence of $H$ and $B$ atoms is totally different: we can see that main defects are $\mathrm{VX}_{m}$ clusters (see Fig. 9 and 10). However, the consequences depend on the specie. B atoms always prefer to regroup in $\mathrm{VB}_{m}$ clusters. For $\mathrm{H}$ atoms, according to $\mathrm{T}$, we obtain different results as we will see below.

More precisely, at low temperature, $\mathrm{C}_{6}$ clusters are the main defects even at undetectable concentrations (see Fig. 8). When T increases, the transition (in $C_{\text {tot }}[C]$ ) between large clusters and the free state decreases. We can therefore expect that at high $\mathrm{T}$ and experimental concentration ( $<100 \mathrm{appm})$, clusters are dissolved. Hence, $C$ atoms should be located only in $o$ sites. In addition, despite negative values of segregation energy for some $\mathrm{VC}_{m}$ clusters, the high stability of $C_{m}$ implies that $C$ atoms will preferentially form clusters without a vacancy.

These results should be looked at carefully. In our statistical model, at low temperature and for high values of $C_{\text {tot }}[X]$, the major clusters are the largest $X_{m}$ clusters considered by the model, so that all carbon atoms are located in these clusters. We reach here the limitations of our approach. At this level of approximation, only small clusters are included: those composed of 6 or fewer atoms, see Fig. 8. We have developed a general statistical model that can include all cluster sizes, but in the first-order approximation and due to the constraints of our approach, we only considered small clusters. The case of $\mathrm{C}$ atoms (small clusters) points out the limits of our approximation. If we wanted to improve the physical description of our system, we should adopt an approach including clusters of all sizes. However, these results allow for a reasonable assumption stating that the limit of solubility of these elements is very low. This is consistent with experimental findings: when carbon concentration becomes too "high", $C_{m}$ clusters are formed and C atoms precipitate.

A similar analysis can be made for $\mathrm{N}$ atoms: nitrogen forms $\mathrm{N}_{m}$ clusters only at low T (around $300 \mathrm{~K}$ ) and is free at higher temperatures. Once again, our results state that the nitrogen solubility is low. Our model predicts that for a high concentration of nitrogen, large amounts of $N_{m}$ will be formed, which should lead to the precipitation of AlN.

The physics of oxygen in $\mathrm{Al}$ is completely different from what has been observed in nickel [7] and iron [4]. In these systems, the strong interaction between vacancies and $\mathrm{O}$ atoms induces that $\mathrm{O}$ atoms form clusters even at high $\mathrm{T}$. In $\mathrm{Al}$, we showed that the interaction between $\mathrm{O}$ and vacancies are weak. At high $\mathrm{T}$ (above $600 \mathrm{~K}$ and at experimental concentrations), we therefore found that $\mathrm{O}$ atoms are located in tetrahedral sites. There are no clusters with vacancies. At low temperature, $O$ concentration is significantly low and $\mathrm{O}$ atoms are to be located in interstitial position only. However, unlike what was found for $\mathrm{C}$ atoms, and even though it is difficult to extrapolate the formation energies of large $\mathrm{O}_{m}$ clusters, it is expected that these clusters will not change the relative concentration of the different clusters and modify the final result being that $O$ atoms are mainly located in tetrahedral sites.

For $\mathrm{H}$ and $\mathrm{B}$ atoms, one expects that the concentration of clusters with vacancies will become not negligible, see Fig. 9. In the case of hydrogen, above $600 \mathrm{~K}, \mathrm{H}$ atoms remain predominantly alone in interstitial position (see Figs. 7 and 8), i.e. in the tetrahedral 

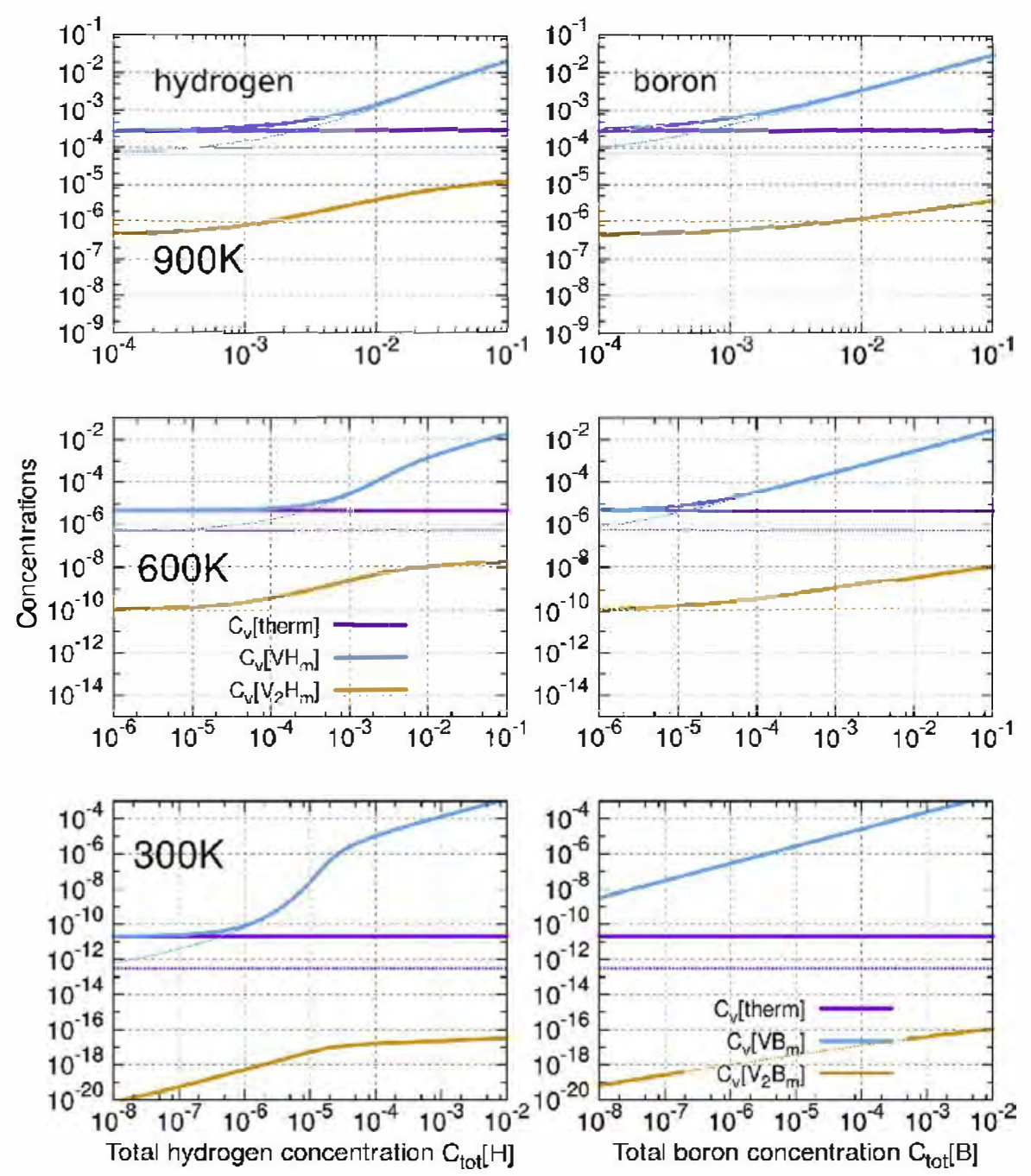

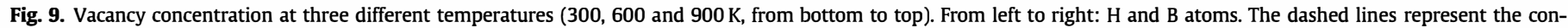
centrations obtained with the experimental value of the vacancy.

position and $\mathrm{VX}_{m}$ are dissolved. When the temperature drops (above $600 \mathrm{~K}$ ), the vacancy concentration increases significantly in the presence of $\mathrm{H}$ atoms, even though most of $\mathrm{H}$ atoms remain free, exactly what is observed in nickel [1,31]. Nonetheless, in these conditions, while the concentration of $\mathrm{VH}_{m}$ clusters is many orders of magnitude higher than thermal concentrations, these larger clusters do not influence the total concentrations of defects. At intermediate temperatures $(600 \mathrm{~K})$ and experimental $\mathrm{H}$ concentrations ( $<1000 \mathrm{appm}$ ), $\mathrm{VH}_{1}$ and $\mathrm{VH}_{2}$ are the main clusters (Fig. 10). Their concentrations are 1-2 orders of magnitude higher than thermal vacancy concentration. At low $\mathrm{T}(300 \mathrm{~K})$, there is a change as we can see on Fig. 7. In the range of $\left[10^{-6} ; 10^{-4}\right] \mathrm{H}$ concentration, the amount of clusters increases significantly, to reach a maximum (above $10^{-4}$ ) when all $\mathrm{H}$ atoms are located in $\mathrm{VH}_{n}$ clusters (see Figs. 7 and 9). In this case the nature of the predominant defects depends on the hydrogen concentration (see Fig. 10): from the $\mathrm{VH}_{1}$ cluster for low concentrations $\left(10^{-6}\right.$, i.e. 1 appm) to $\mathrm{VH}_{6}$ for high concentrations $\left(10^{-3}\right.$, i.e. $1000 \mathrm{H}$ appm). This result agrees with earlier results [5].

If we include multi-vacancy clusters, the concentration of $\mathrm{V}_{2} \mathrm{H}_{m}$ clusters remains always many orders of magnitude lower than the concentration of $\mathrm{VH}_{m}$ defects and can therefore be neglected (see Fig. 9). The maximal effect is at low $\mathrm{T}(300 \mathrm{~K})$, where full filled vacancies $\left(\mathrm{VH}_{6}\right)$ have large concentration for usual $\mathrm{H}$ concentrations. We clearly see that even if $\mathrm{VH}_{m}$ should move slowly like in nickel [33], the abundance of vacancies will affects the diffusion of species in aluminum.

In the case of B atoms, the physics is different. We note that $C\left[V B_{m}\right]$ is higher than those of other clusters, and especially of free atoms, see Fig. 9. $B$ atoms always aggregate and form clusters with a vacancy (see Fig. 7). A small content of B atoms therefore induces an important vacancy concentration, the same order of magnitude than B concentration. As suggested by our DFT values, one notes that main clusters are the $\mathrm{VB}_{3}$ and $\mathrm{VB}_{4}$.

\section{Conclusion}

In conclusion, we investigated the solubility of carbon, nitrogen, hydrogen, boron and oxygen in aluminum by using a multi-scale approach. DFT simulations of isolated atoms and clusters enabled us to: (i) show that $\mathrm{C}$ and $\mathrm{B}$ atoms prefer to be located in octahedral sites, while $\mathrm{O}, \mathrm{H}$ and $\mathrm{N}$ atoms prefer tetrahedral sites. The insertion of species in such positions (Bader charge, elastic dipole, solubility energies ...) within the metal have been fully characterized. (ii) In the case of $C$ atoms, we identified one new position, which is nevertheless less stable than the octahedral site. $O$ and $B$ atoms 

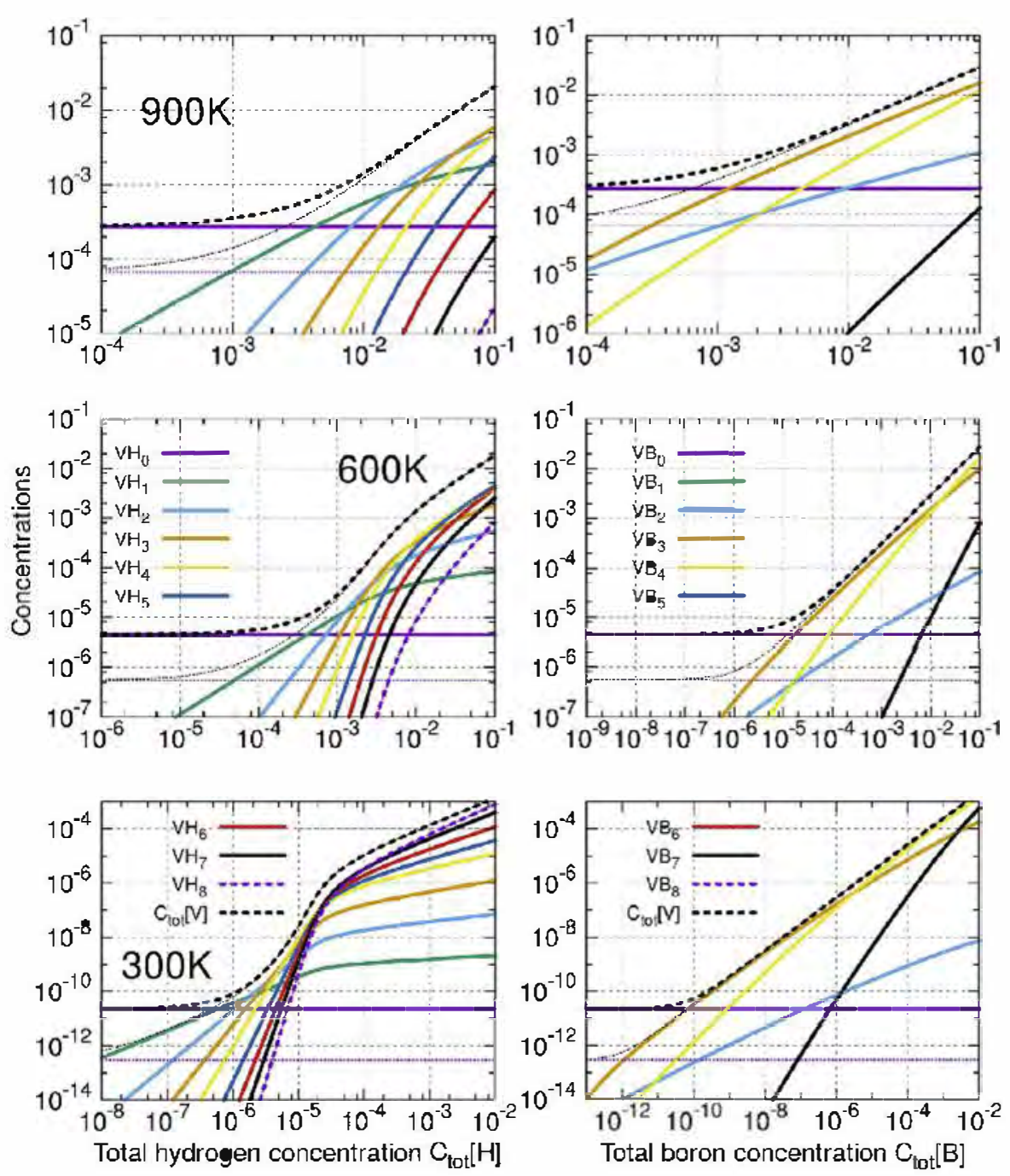

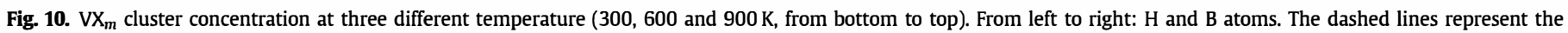
concentrations obtained with the experimental value of the vacancy. The thick dashed lines are the total vacancy concentrations $C_{\text {tot }}[V]$.

only have one stable position each: $t$ and $o$ sites, respectively. (iii) From the study of interactions $\left(\mathrm{X}-\mathrm{X}, \mathrm{V}-\mathrm{X}\right.$ and $\left.\mathrm{V}_{2} \mathrm{X}\right)$, including large clusters, we see that some clusters are stable either under the form of $X_{m}$ (in the case of $\mathrm{C}, \mathrm{N}$ and $\mathrm{O}$ atoms) or $\mathrm{VX}_{m}$ clusters ( $\mathrm{H}$ and $\mathrm{B}$ atoms). Pair interaction energies are also reported, directly useful for a Hamiltonian approach. These data characterize interactions between the metal and interstitial elements.

To capture the thermal effects, we used a statistical approach that includes DFT energies of the different types of clusters: $X_{m}$, $V X_{m}$ and $V_{2} X_{m}$. We show that at high temperature $\mathrm{C}, \mathrm{N}, \mathrm{O}$ and $\mathrm{H}$ atoms are in interstitial positions. Our results show that these atoms do not modify significantly the vacancy concentration, contrary to what was found for B atoms. At low T, the physics changes: for carbon, oxygen and nitrogen form preferentially $X_{m}$ clusters, whereas hydrogen atoms are in $t$ sites but $\mathrm{VH}_{m}$ concentrations are high. Regarding $B$ atoms, they always form clusters with a vacancy.

\section{Acknowledgments}

This work was performed using HPC resources from CALMIP (Grant 2016-p0842, 2016-p0749 and 2017-p0749).

\section{Appendix A. References states and alloys of Al}

\section{Appendix A.1. Diamond and boron structures}

To evaluate the Helmholtz energy of carbon diamond, we calculated the inter-atomic force constants using the finite displacement approach. They were calculated displacing only non equivalent atoms along non equivalent directions according to the symmetry of the system. The phonopy package [23] was used to generate finite displacements according to the symmetry of each structure. It was then used to analyze and compute vibrational properties. A $3 \times 3 \times 3$ super-cell ( 54 atoms in the unit-cell) was used and only one finite displacement is necessary for diamond.

In the case of boron, we used the hR12 structure, space group No 166. There are two types of atoms B-I and B-II that occupy the same $(6 \mathrm{~h})$ sites $\left(u_{1}, u_{1}, u_{2}\right)$. This structure was used as a reference state, even the $\beta$-B [34] (space group No 166) and the B t-50 (space group No 134 , where B atoms are in $2 b, 8 m, 8 m, 16 n$ and $16 n$ ) structures are often referred as more stable. Table A.1 summarizes these results. 
Table A.1

Formation energies ( $E_{f}$, in eV/atom), lattice parameters (in $\AA$ ) of different Al-compounds.

\begin{tabular}{|c|c|c|c|c|c|}
\hline structure & & $a_{0}$ & $c_{0}$ & $u_{i}$ & $E_{f}$ \\
\hline \multirow[t]{2}{*}{$\mathrm{Al}$ (fcc) } & & 4.04 & - & - & 0 \\
\hline & [35] & 4.04 & - & - & - \\
\hline \multirow[t]{2}{*}{$\mathrm{C}$ (diamond) } & & 3.574 & - & - & 0 \\
\hline & [35] & 3.567 & - & - & - \\
\hline \multirow[t]{2}{*}{$\mathrm{B}(\alpha)$} & & 2.828 & 4.182 & $0.010 / 0.654$ & 0 \\
\hline & & & & $0.221 / 0.630$ & \\
\hline \multirow[t]{2}{*}{$\mathrm{B}(\beta)$} & & 11.073 & 23.804 & - & 0.135 \\
\hline & [18] & 10.925 & 23.814 & - & - \\
\hline$B(t-50)$ & & 8.838 & 4.952 & - & 0.097 \\
\hline \multirow[t]{3}{*}{ WZ } & $\mathrm{AlO}$ & 3.689 & 6.101 & 0.350 & -1.684 \\
\hline & AlN & 3.128 & 5.015 & 0.381 & -1.413 \\
\hline & [13] & 3.111 & 4.979 & - & - \\
\hline \multirow[t]{2}{*}{ ZB } & $\mathrm{AlO}$ & 2.266 & - & - & -1.711 \\
\hline & AlN & 2.198 & - & - & -1.392 \\
\hline \multirow[t]{2}{*}{$\mathrm{NaCl}$} & AlC & 4.268 & - & - & +0.421 \\
\hline & AlB & 4.542 & - & - & +0.623 \\
\hline \multirow[t]{2}{*}{$\mathrm{Al}_{2} \mathrm{O}_{3}$} & & 5.177 & $55^{\circ} 29$ & $0.352 / 0.943$ & -3.026 \\
\hline & [14] & 5.127 & $55^{\circ} 16$ & - & - \\
\hline \multirow[t]{4}{*}{$\mathrm{Al}_{4} \mathrm{C}_{3}$} & & 3.353 & 25.117 & $0.129 / 0.293$ & -0.152 \\
\hline & & & & 0.217 & \\
\hline & [36] & 3.335 & 24.059 & $0.129 / 0.294$ & - \\
\hline & & & & 0.217 & \\
\hline \multirow[t]{2}{*}{$\mathrm{AlB}_{2}$} & & 3.008 & 3.288 & - & -0.043 \\
\hline & [18] & 3.006 & 3.252 & - & - \\
\hline
\end{tabular}

\section{Appendix A.2. Aluminum alloys}

Finally, we conducted additional calculations on several Al-X alloys: the wurtzite ( $\mathrm{WZ}, \mathrm{hP} 4$, space group $\left.\mathrm{PG}_{3} m c\right)$, where $\mathrm{Al}$ atoms are located in $2 b(1 / 3,2 / 3,0)$ and $X$ in $2 b(1 / 3,2 / 3, u)$, the zincblende structure $(\mathrm{ZB}, \mathrm{CF} 8$, space group $F \overline{4} 3 \mathrm{~m})$ where $\mathrm{Al}$ atoms are in $4 a(0,0,0)$ and $\mathrm{X}$ in $4 c(1 / 4,1 / 4,1 / 4)$ and the $\mathrm{NaCl}$ structure (cF8, space group $F m \overline{3} m$ ) where $\mathrm{Al}$ atoms are in $4 a(0,0,0)$ and $\mathrm{X}$ in $4 b(0.5,0.5,0.5)$. We also reported results for additional structures: (i) the corundum $\mathrm{Al}_{2} \mathrm{O}_{4}$ structure (hR10, space group $R \overline{3} c$ ), where $\mathrm{Al}$ atoms are in $4 c\left(u_{1}, u_{1}, u_{1}\right)$ and $\mathrm{O}$ in $6 e\left(u_{2}, 1 / 2-u_{2}, 1 / 4\right)$; (ii) the $\mathrm{Al}_{4} \mathrm{C}_{3}$ structure (hR7, space group $R \overline{3} m)$ where $\mathrm{Al}$ atoms are in two $6 c(0$, $\left.0, \mathrm{u}_{1,2}\right)$ and $\mathrm{C}$ in $6 c\left(0,0, \mathrm{u}_{3}\right)$ and $3 a(0,0,0)[36]$; and (iii) the $\mathrm{AlB}_{2}$ structure ( $\omega$ phase, $h P 3$, space group $P 6 / \mathrm{mmm}$ ), where $B$ atoms are in $2 d(1 / 3,2 / 3,1 / 2)$ and $\mathrm{Al}$ in $1 a(0,0,0)$.

The $\mathrm{NaCl}$ structure corresponds to an fcc structure in which all octahedral sites are occupied. The AlX zincblende is an fcc-Al where half of the tetrahedral sites are occupied. Table A.1 summarizes these results. DFT results are in excellent agreement with experimental values (lattice parameters and Wyckoff positions) for AlN wurtzite, $\mathrm{Al}_{2} \mathrm{O}_{3}, \mathrm{Al}_{4} \mathrm{C}_{3}$ and $\mathrm{AlB}_{2}$. One can notice that experimental structures are always the more stable structures.

\section{References}

[1] D. Tanguy, Y. Wang, D. Connétable, Stability of vacancy-hydrogen clusters in nickel from first principles calculations, Acta Mater. 78 (2014) 135-143.

[2] Y. Wang, D. Connétable, D. Tanguy, Influence of trap connectivity on h diffusion: vacancy trapping, Acta Mater. 103 (2016) 334-340.

[3] C. Barouh, T. Schuler, C.-C. Fu, M. Nastar, Interaction between vacancies and interstitial solutes (c, n, and o) in $\alpha$-fe: from electronic structure to thermodynamics, Phys. Rev. B 90 (2014) 054112.

[4] T. Schuler, C. Barouh, M. Nastar, C.-C. Fu, Equilibrium vacancy concentration driven by undetectable impurities, Phys. Rev. Lett. 115 (2015) 015501, https:// doi.org/10.1103/PhysRevLett.115.015501 http://link.aps.org/doi/10.1103/ PhysRevLett.115.015501.

[5] D. Tanguy, $\mathrm{H}$ effects in al-mg, al-zn-mg alloys, and al: experiments, continuum, and atomistic modeling, Corrosion 72 (2) (2016) 297-313, https:// doi.org/10.5006/1854. https://doi.org/10.5006/1854.

[6] A. Sieverts, The absorption of gases by metals, Z. Metallkd 21 (1929) 37-46.

[7] D. Connétable, M. David, A. Prillieux, D. Young, D. Monceau, Impact of the clusterization on the solubility of oxygen and vacancy concentration in nickel: a multi-scale approach, J. Alloys Compd. 708 (2017) 1063-1072. https://doi. org/10.1016/j.jallcom.2017.03.018.

[8] R. Nazarov, T. Hickel, J. Neugebauer, $A b$ initio study of h-vacancy interactions in fcc metals: implications for the formation of superabundant vacancies, Phys. Rev. B 89 (2014) 144108, https://doi.org/10.1103/PhysRevB.89.144108. http://link.aps.org/doi/10.1103/PhysRevB.89.144108.

[9] G. Lu, E. Kaxiras, Hydrogen embrittlement of aluminum: the crucial role of vacancies, Phys. Rev. Lett. 94 (2005) 155501, https://doi.org/10.1103/PhysRevLett.94.155501. http://link.aps.org/doi/10.1103/PhysRevLett.94.155501.

[10] M. Ji, C.-z. Wang, K.-m. Ho, S. Adhikari, K.R. Hebert, Statistical model of defects in al-h system, Phys. Rev. B 81 (2010) 024105, https://doi.org/10.1103/PhysRevB.81.024105. http://link.aps.org/doi/10.1103/PhysRevB.81.024105.

[11] L. Yang, X. Zu, F. Gao, Ab initio study of formation, migration and binding properties of helium-vacancy clusters in aluminum, Phys. B Condens. Matter 403 (17) (2008) 2719-2724. https://doi.org/10.1016/j.physb.2008.02.015.

[12] M. David, D. Connétable, Diffusion of interstitials in metallic systems, illustration of a complex study case: aluminum, J. Phys. Condens. Matter 29 (45) (2017), 455703. http://stacks.iop.org/0953-8984/29/i=45/a=455703.

[13] H.A. Wriedt, The al-n (aluminum-nitrogen) system, Bull. Alloy Phase Diagrams 7 (4) (1986) 329-333, https://doi.org/10.1007/BF02873001. https:// doi.org/10.1007/BF02873001.

[14] H.A. Wriedt, The al-o (aluminum-oxygen) system, Bull. Alloy Phase Diagrams 6 (6) (1985) 548-553, https://doi.org/10.1007/BF02887157. https://doi.org/ $10.1007 / B F 02887157$.

[15] B. Grashchenko, V. Darovskii, Khandzhyan, Tr. Vses. Alyumin. Magnievyi. Inst. 90.

[16] H. Okamoto, A1-c (aluminum-carbon), J. Phase Equil. 13 (1) (1992) 97-98 https://doi.org/10.1007/BF02645389. https://doi.org/10.1007/BF02645389.

[17] A. San-Martin, F. Manchester, The al-h (aluminum-hydrogen) system, J. Phase Equil. 13 (1) (1992) 17-21, https://doi.org/10.1007/BF02645371. https://doi. org/10.1007/BF02645371.

[18] H. Duschanek, P. Rogl, The al-b (aluminum-boron) system, J. Phase Equil. 15 (5) (1994) 543-552, https://doi.org/10.1007/BF02649415. https://doi.org/10. $1007 / B F 02649415$.

[19] G. Kresse, J. Hafner, Ab initio molecular dynamics for liquid metals, Phys. Rev. B 47 (1993) 558R.

[20] J. Perdew, K. Burke, M. Ernzerhof, Generalized gradient approximation made simple, Phys. Rev. Lett. 78 (1997) 1396.

[21] G. Kresse, D. Joubert, From ultrasoft pseudopotentials to the projector augmented-wave method, Phys. Rev. B 59 (1999) 1758.

[22] H. Monkhorst, J. Pack, Special points for the brilluoin zone integrations, Phys. Rev. B 13 (1976) 5188.

[23] A. Togo, F. Oba, I. Tanaka, First-principles calculations of the ferroelastic transition between rutile-type and ca, Phys. Rev. B 78 (2008) 134106.

[24] D. Connétable, E. Andrieu, D. Monceau, First-principles nickel database: energetics of impurities and defects, Comput. Mater. Sci. 101 (2015) 77-87.

[25] C. Wolverton, V. Ozolins, M. Asta, Hydrogen in aluminum: first-principles calculations of structure and thermodynamics, Phys. Rev. B 69 (2004) 144109.

[26] A. Glensk, B. Grabowski, T. Hickel, J. Neugebauer, Breakdown of the arrhenius law in describing vacancy formation energies: the importance of local anharmonicity revealed by $A b$ initio thermodynamics, Phys. Rev. X 4 (2014) 011018, https://doi.org/10.1103/PhysRevX.4.011018. http://link.aps.org/doi/ 
10.1103/PhysRevX.4.011018.

[27] H. Wang, D. Rodney, D. Xu, R. Yanga, P. Veyssière, Pentavacancy as the key nucleus for vacancy clustering in aluminum, Phys. Rev. B 84 (2011) 220103.

[28] J. Dai, Y. Song, Influence of h, c, $\mathrm{n}$ and o impurities on the stability of mg and a from first-principles calculations, Model. Simulat. Mater. Sci. Eng. 21 (5) (2013) 055014. http://stacks.iop.org/0965-0393/21/i=5/a=055014.

[29] E. Clouet, S. Garruchet, H. Nguyen, M. Perez, C. Becquart, Dislocation interaction with in alpha-fe: a comparison between atomic simulations and elasticity theory, Acta Mater. 56 (2008) 3450-3460.

[30] D. Tanguy, Y. Wang, D. Connétable, Stability of vacancy-hydrogen clusters in nickel from first principles calculations, Acta Mater. 78 (2014) 135-143.

[31] D. Connétable, Y. Wang, D. Tanguy, Segregation of hydrogen to defects in nickel using first-principle calculations: the case of self-interstitials and cavities, J. Alloys Compd. 614 (2014) 211-220.

[32] C. Domain, C.S. Becquart, J. Foct, $A b$ initio study of foreign interstitial atom (c,n) interactions with intrinsic point defects in $\alpha$-fe, Phys. Rev. B 69 (2004) 144112,
https://doi.org/10.1103/PhysRevB.69.144112. http://link.aps.org/doi/10.1103/ PhysRevB.69.144112.

[33] Y. Wang, D. Connétable, D. Tanguy, Hydrogen influence on diffusion in nickel from first-principles calculations, Phys. Rev. B 91 (2015) 094106.

[34] B. Callmer, An accurate refinement of the $\beta$-rhombohedral boron structure, Acta Crystallogr. Sect. B 33 (6) (1977) 1951-1954, https://doi.org/10.1107/ S0567740877007389. https://doi.org/10.1107/S0567740877007389.

[35] C. Kittel, Introduction to Solid State Physics, Wiley, New York, 1996.

[36] P. Villars, K. Cenzual, J. Daams, R. Gladyshevskii, O. Shcherban, V. Dubenskyy, N. Melnichenko-Koblyuk, O. Pavlyuk, I. Savysyuk, S. Stoyko, L. Sysa, Structure Types. Part 5: Space Groups (173) P63- (166) R-3m Al4c3: Datasheet from Landolt-börnstein - Group iii Condensed Matter Volume 43a5: "structure Types. Part 5: Space Groups (173) P63-(166) R-3m' in Springermaterials (https://doi.org/10.1007/978-3-540-46933-9_435), copyright 2007 SpringerVerlag. doi: 10.1007/978-3-540-46933-9_435. URL http://materials.springer. com/lb/docs/sm_lbs_978-3-540-46933-9_435. 